\title{
Novel Intronic RNA Structures Contribute to Maintenance of Phenotype in Saccharomyces cerevisiae
}

\author{
Katarzyna B. Hooks, ${ }^{1,2}$ Samina Naseeb, ${ }^{2}$ Steven Parker, Sam Griffiths-Jones, ${ }^{3}$ and Daniela Delneri ${ }^{3}$
}

Faculty of Life Sciences, University of Manchester, M13 9PT, United Kingdom

\begin{abstract}
The Saccharomyces cerevisiae genome has undergone extensive intron loss during its evolutionary history. It has been suggested that the few remaining introns (in only $5 \%$ of protein-coding genes) are retained because of their impact on function under stress conditions. Here, we explore the possibility that novel noncoding RNA structures (ncRNAs) are embedded within intronic sequences and are contributing to phenotype and intron retention in yeast. We employed de novo RNA structure prediction tools to screen intronic sequences in S. cerevisiae and 36 other fungi. We identified and validated 19 new intronic RNAs via RNA sequencing (RNA-seq) and RT-PCR. Contrary to the common belief that excised introns are rapidly degraded, we found that, in six cases, the excised introns were maintained intact in the cells. In another two cases we showed that the ncRNAs were further processed from their introns. RNA-seq analysis confirmed that introns in ribosomal protein genes are more highly expressed when they contain predicted RNA structures. We deleted the novel intronic RNA structure within the GLC7 intron and showed that this region, rather than the intron itself, is responsible for the cell's ability to respond to salt stress. We also showed a direct association between the in cis presence of the intronic RNA and GLC7 expression. Overall, these data support the notion that some introns may have been maintained in the genome because they harbor functional RNA structures.
\end{abstract}

KEYWORDS introns; ncRNA; RNA structure; yeast

T HERE are three main theories regarding the origin of introns: "Introns Late," "Introns Early," and "Introns First" (Jeffares et al. 2006). The Introns Late and Introns Early theories suggest that introns arose within the eukaryotic lineage, and before the Prokaryota-Eukaryota split, respectively, whereas Introns First implies that these noncoding sequences appeared before protein-coding genes. Introns are maintained, lost or gained with different rates within different eukaryotic lineages. The recent evolution of the yeast genome has seen widespread

Copyright @ 2016 Hooks et al.

doi: 10.1534/genetics.115.185363

Manuscript received November 26, 2015; accepted for publication May 13, 2016; published Early Online May 18, 2016.

Available freely online through the author-supported open access option.

This is an open-access article distributed under the terms of the Creative Commons Attribution 4.0 International License (http://creativecommons.org/licenses/by/4.0/), which permits unrestricted use, distribution, and reproduction in any medium, provided the original work is properly cited.

Supplemental material is available online at www.genetics.org/lookup/suppl/doi:10. 1534/genetics.115.185363/-/DC1.

${ }^{1}$ Present address: U1035 INSERM, Université de Bordeaux, 33076 Bordeaux Cedex, France.

${ }^{2}$ These authors contributed equally to this work.

${ }^{3}$ Corresponding authors: Faculty of Life Sciences, University of Manchester, Michael Smith Bldg., Oxford Rd., Manchester, M13 9PT, UK. E-mail: d.delneri@manchester.ac. uk; and Faculty of Life Sciences, University of Manchester, Michael Smith Bldg., Oxford Rd. Manchester, M13 9PT, UK. E-mail: sam.griffiths-jones@manchester.ac.uk intron loss, with the result that only $5 \%$ of Saccharomyces cerevisiae genes contain introns (Dujon 2006). It has been proposed that small organisms with a large effective population size, such as $S$. cerevisiae, have seen deleterious introns gradually eliminated from the genome (Lynch 2002). This raises the question of why some introns are maintained in the yeast genome and, furthermore, whether they are functionally relevant.

Recent studies indicate that $S$. cerevisiae introns increase fitness under stress (Parenteau et al. 2008), aid ribosome assembly, and regulate expression of the paralogous copy of the gene (Parenteau et al. 2011). Introns may contain regulatory sequences and structures that affect splicing or expression of their host genes. In the HAC1 transcript, short RNA hairpins define the intron boundaries (Sidrauski and Walter 1997). Splicing of the YRA1 messenger RNA (mRNA) regulates its export from the nucleus (Preker et al. 2002; Rodriguez-Navarro et al. 2002). Described RNA structures in pre-mRNA transcripts of RPL30 (Li et al. 1996) and RPS14B (Fewell and Woolford 1999) regulate splicing of their host genes. Intronic hairpins in RPL18A and RPS22B pre-mRNAs are recognized by RNase III Rnt1p and promote mRNA degradation (Danin-Kreiselman et al. 2003). The RPS17B intron contains a RNA-based splicing enhancer that physically decreases the distance between the 
splice sites (Rogic et al. 2008). RNA structures in the introns of $R P S 9 A$ and RPS9B transcripts are vital components of an autoregulatory circuit (Plocik and Guthrie 2012).

The cases described above are examples where introns function in cis; intronic sequences can also act in trans. In vertebrates, introns frequently harbor functional noncoding RNAs (ncRNAs). Tiling arrays and deep RNA sequencing (RNA-seq) have revealed the existence of many novel intronic transcripts, most of which have no known function (Cheng et al. 2005; Mercer et al. 2008). In addition, a recent study searching for RNAs bound by the chromatin-modifying polycomb complex raised the possibility that intronic ncRNAs can be used to guide chromatin modifications that influence gene expression in a manner analogous to some long intergenic ncRNAs (Guil et al. 2012). In vertebrates, there are also well-characterized examples of intronic ncRNAs such as transfer RNAs (tRNAs), small nucleolar RNAs (snoRNAs), and microRNAs (miRNAs) (Kim and Kim 2007). However, in $S$. cerevisiae, well-known intronic ncRNAs classes are not prevalent: miRNAs are nonexistent, due to the loss of pre-miRNA processing enzymes (Drinnenberg et al. 2009); intronic snoRNAs have been mostly "deintronized" (Mitrovich et al. 2010); and all tRNAs are present outside introns. Even though many ncRNAs have been found in $S$. cerevisiae, such as stable unannotated transcripts (SUTs) and cryptic unstable transcripts (CUTs) (Wu et al. 2012), only 2\% of the instances of these classes overlap with introns ( $\mathrm{Xu}$ et al. 2009). It is commonly believed that splicing in higher eukaryotes increases protein diversity by providing multiple mRNAs from a single locus. However, alternative splicing in S. cerevisiae has been shown only for transcripts from the genes SRC1 (Davis et al. 2000; Grund et al. 2008), PTC7 (Juneau et al. 2009), and MTR2 (Davis et al. 2000; Preker et al. 2002). Since alternative splicing in $S$. cerevisiae is rare and there are few typical intronic ncRNAs, we hypothesize that functional yeast introns may have been retained because they contain novel ncRNAs or pre-mRNA structures.

In order to discover potential functional RNA structures within introns in $S$. cerevisiae, we performed a computational screen for novel structures using intron orthologs from 36 fungal species and employing three de novo RNA structure prediction tools. The screen identified 19 introns containing potential RNA structures, and we validated the expression and processing of a subset by RT-PCR. We showed that six introns tested are maintained in the cell after splicing and two contain candidate novel ncRNAs. A novel RNA structure within the GLC7 intron, rather than the whole intron (Juneau et al. 2006; Parenteau et al. 2008), is responsible for the cell's ability to respond to salt stress, by altering the gene expression.

\section{Materials and Methods}

\section{Intron alignments}

Sequences of intron-containing genes were extracted from the Saccharomyces Genome Database (SGD, http://www.yeastgenome.org/). Genes orthologous to intron-containing genes from $S$. cerevisiae were identified in 36 fungal genome sequences (Supplemental Material, Table S1) using TBLASTX, with the coding gene sequence as the query and with the following settings: -E e-6 -qframe 1 -hspsepsmax 1000 -topcomboN 1 . We collected the sequences of putative orthologs with at least $65 \%$ query coverage, to which $1000 \mathrm{bp}$ and $300 \mathrm{bp}$ of flanking sequence were added to the $5^{\prime}$ and $3^{\prime}$ ends of each hit. The putative ortholog gene sequences were then searched for the presence of the orthologous intron using BLASTN with options: -E 0.1 -W 3 -hspsepsmax 1000. The best hit (with the lowest $e$-value and confirmed by manual inspection) was retained for each intron in each species.

\section{RNA structure predictions}

Three structure prediction programs were used: CMfinder (v 0.2), RNAz (v 2.0), and Evofold (v 7b). Sequences of orthologous introns were used for predictions with CMfinder as described in Torarinsson et al. (2008). CMfinder was run with settings: -n 5 -m 30 -M 100 and -s 2 -n 5 -m 40 -M 100, and identified motifs were extended using the CombMotif.pl procedure. Motifs with a composite score of $r>5$ and folding free energy of $<-5 \mathrm{kcal} \cdot \mathrm{mol}^{-1}$ were considered as putative positives. For RNAz and EvoFold predictions, intron sequences were first aligned with mLAGAN. Structure prediction by RNAz was performed according to the manual (http://www.tbi.univie.ac.at/ wash/RNAz/manual.pdf). The, rnazWindow.pl script was used to slice the alignments with the following options: - max-gap $=0.25-\min -\mathrm{id}=30-$ maxseqs $=6$. RNAz was then run on the forward strand of the gapped alignments (options: -forward -g - p 0) and sequences with probability $P>0.5$ were considered as putative positives. For EvoFold predictions, the required phylogenetic tree containing the species present in the intron alignments was derived by pruning the tree presented by Medina et al. (2011), and the subsequent structure predictions were predicted using default parameters. We employed a threshold value of 10 for the log-odds ratio of the likelihood of the region under the structure model and background model. The complete list of all predictions can be found in File S1. To extend the phylogenetic range of the RNA predictions, BLAST and INFERNAL 1.0.2 (Nawrocki et al. 2009) were used to re-search all fungal genomes in an iterative process, based on the Rfam approach (Gardner et al. 2010) and as described previously (Hooks and Griffiths-Jones 2011).

\section{Strains and media}

Intron sequence replacement strains were engineered from the BY4743 (MATa/ $\alpha$, his $3 \Delta 1 /$ his $3 \Delta 1$, leu $2 \Delta 0 /$ leu $2 \Delta 0$, lys2$\Delta 0 / L Y S 2$, MET15/met15 $\Delta 0$, and ura3 $\Delta 0 /$ ura3 $\Delta 0$ ), BY4742 $(M A T \alpha$, his3 $\Delta 1$, leu $2 \Delta 0$, lys $2 \Delta 0$, and ura3 $\Delta 0$ ) and BY4741 (MATa, his $3 \Delta 1$, leu $2 \Delta 0$, met $15 \Delta 0$, and ura3 $\Delta 0$ ) parental strains and maintained in YPD medium containing $2 \%$ $(\mathrm{w} / \mathrm{v})$ yeast extract, $1 \%(\mathrm{w} / \mathrm{v})$ peptone, and $2 \%(\mathrm{w} / \mathrm{v})$ glucose. The transformants were plated on solid YPD medium with $300 \mathrm{mg} / \mathrm{ml}$ geneticin (Gibco BRL) for kanMX selection and on $10 \mathrm{mg} / \mathrm{ml}$ phleomycin (Invitrogen) for pCre-ble 
selection. Mineral salts medium (F1 medium) used was of the following composition (grams/liter); $\left(\mathrm{NH}_{4}\right)_{2} \mathrm{SO}_{4}$ (3.13), $\mathrm{KH}_{2} \mathrm{PO}_{4}(2), \mathrm{MgSO}_{4} \cdot 7 \mathrm{H}_{2} \mathrm{O}(0.55), \mathrm{NaCl}(0.1), \mathrm{CaCl}_{2} \cdot 2 \mathrm{H}_{2} \mathrm{O}$ (0.09), and $2 \mathrm{ml}$ of trace element solution per liter was added to it. The trace element solution used was of the following composition (grams/liter): $\mathrm{ZnSO}_{4} \cdot 7 \mathrm{H}_{2} \mathrm{O}(0.7), \mathrm{CuSO}_{4} \cdot 5 \mathrm{H}_{2} \mathrm{O}$ $(0.1), \mathrm{H}_{3} \mathrm{BO}_{3}(0.1)$, and $\mathrm{KI}(0.1)$. The F1 was supplemented with $2 \%(\mathrm{w} / \mathrm{v})$ glucose and $1.65 \mathrm{ml}$ vitamin stock before use. The synthetic minimal SD medium $(0.67 \%$ Bacto yeast nitrogen base without amino acids, $2 \%$ glucose) and F1 medium were also supplemented with required amino acids appropriate for the parental strain (Baganz et al. 1998). The media were filter sterilized.

\section{RNA extraction}

The $S$. cerevisiae strain BY4741 was grown in $500 \mathrm{ml}$ rich media (YPD) in $30^{\circ}$ with shaking at $200 \mathrm{rpm}$ to an absorbance of 0.5 at $600 \mathrm{~nm}$. The RNA was extracted using Trizol (Invitrogen), precipitated in lithium chloride (Ambion), washed twice with 70\% ethanol, and the pellet resuspended in $\mathrm{dH}_{2} \mathrm{O}$. RNA concentration and quality was evaluated by measuring absorbance at $260 \mathrm{~nm}$ on a NanoDrop spectrometer ND-1000 (Thermo Scientific). The low molecular weight enriched RNA sample was obtained from total RNA as described in Catalanotto et al. (2002). Total RNA was used for RNA-seq and both total and low molecular weight enriched RNA was used for RT-PCR.

\section{$R T-P C R$}

Complementary DNA (cDNA) was synthesized from $2 \mu \mathrm{g}$ RNA of either total or low-molecular weight RNA using QuantiTect Reverse Transcription Kit (Qiagen) according to the manufacturer's protocol. Fragments of cDNA corresponding to the predicted intronic RNA structure (ncRNA), whole intron of interest (intron), and exons surrounding the intron (mRNA) were amplified by PCR with BIOTAQ DNA Polymerase (Bioline) according to the supplier's guidelines. The list of all primer sequences used can be found in File S2. The reaction mix was composed of 4 pmol of each primer and $20 \mathrm{ng}$ of total or low molecular weight cDNA for each $10 \mu \mathrm{l}$ of total reaction mixture. The cycling conditions were an initial denaturation for $5 \mathrm{~min}$ at $95^{\circ}, 35$ cycles of denaturation ( $45 \mathrm{sec}, 94^{\circ}$ ), annealing ( $45 \mathrm{sec}$, $56^{\circ}$ ), and elongation ( $90 \mathrm{sec}, 72^{\circ}$ ), followed by a final elongation for $5 \mathrm{~min}$ at $72^{\circ}$. Amplification of both cDNA using snR44 primers was used as a positive control. Genomic DNA extracted from BY4741 with Wizard Genomic DNA Purification Kit (Promega), according to the manufacturer's protocol, was used as a positive control and water was used as a negative control. The PCR products were visualized by ethidium bromide staining on $1.2-3.5 \%$ agarose gels. For each predicted RNA structure, at least two independent PCR reactions with genomic DNA, total cDNA, low molecular weight cDNA, and snR44 positive control primers were performed in order to confirm expression.

\section{Northern hybridization}

A total of $10 \mu \mathrm{g}$ of the total RNA and $10 \mathrm{pmol}$ of the oligonucleotides mimicking the intronic region of GLC7 were loaded in RNA loading dye (Fermentas) onto separate lanes of a denaturating gel containing $36.5 \mathrm{mM}$ MOPS, $9.1 \mathrm{mM}$ sodium acetate, $0.9 \mathrm{mM}$ EDTA, $2 \mathrm{M}$ formaldehyde, $0.5 \mu \mathrm{g} / \mathrm{ml}$ ethidium bromide, and 1\% agarose. RNA transfer, UV crosslinking, and Northern blotting were performed as previously described (Naseeb and Delneri 2012). [ ${ }^{32}$ P]-ATP end-labeled mixtures of two sense (to detect transcription from antisense strand) and two antisense oligonucleotides (to detect transcription from sense strand; all listed in File S2) were used as probes.

\section{Real-time PCR}

The expression levels of the GLC7 gene in the intron replacement mutant and BY4742 wild type grown in F1 media and in $\mathrm{F} 1+0.9 \mathrm{M} \mathrm{NaCl}$ were assessed by quantitative real-time PCR using the QuantiTect real time PCR kit (Qiagen, no. 204143). cDNA was extracted using QuantiTect Reverse Transcription Kit (Qiagen, no. 205311) according to the manufacturer's manual. Real-time primers are listed in File S2. The PCR reactions were performed in triplicate for two independent biological replicas, as described previously (Naseeb and Delneri 2012). Relative normalized fold expression was calculated according to the $\Delta \Delta \mathrm{Ct}$ method using ACT1 as a reference gene.

\section{RNA-seq}

We used our previously generated RNA-seq data, deposited in Gene Expression Omnibus (GEO) under accession no. GSE58884 (Hooks et al. 2014). A total of 77,286,181 50-bp reads were filtered using the approach of Sasson and Michael (2010). Filtering left 45,520,779 reads with an average quality of $>20$, which were mapped to the $S$. cerevisiae genome (SacCer3) using Bowtie with settings -m 1 -v 2 (Langmead et al. 2009; Trapnell et al. 2009). A total of 25,254,315 reads with a maximum of two mismatches were mapped to the $S$. cerevisiae genome. To calculate average number of reads per intron [or the coding sequence (CDS)] [in reads per kilobase per million mapped reads (RPKM)], reads for each intron or CDS were summarized using featureCounts (Liao et al. 2014) and divided by the length of the feature in kilobases and the million reads mapped. The number of reads mapping to introns and their host genes is presented in File S3.

\section{Analysis of exosome target data}

The cross-linking and analysis of cDNA (Complex Reads Analysis and Classification, CRAC) data presented by Schneider et al. (2012) were filtered for genes that contain introns. From the RNAseq data presented here, we calculated the number of reads in RPKM corresponding only to the ORFs for the same set of genes. For each individual CRAC experiment, the number of reads for each gene was normalized by the number of ORF reads for this gene from our RNA-seq data. The percentile rank of normalized values was calculated for each gene in each CRAC experiment, and then averaged across the 16 CRAC experiments (File S3). We considered genes with an average percentile rank with the 
top $10 \%$ to be preferentially bound by the exosome protein components.

\section{Deletion of predicted intronic RNA}

In order to generate deletions of intron fragments or insertions into introns, the Cre-loxP system was used with a kanMX cassette flanked by loxP sites (Guldener et al. 1996). Deletion cassettes were amplified as described previously (Delneri et al. 2003). The $S$. cerevisiae BY4743 strain was transformed with $1 \mu \mathrm{g}$ of each PCR product according to Gietz and Schiestl (2007). Selection of mutants and PCR confirmation were performed as described by Carter and Delneri (2010). The strains with the loxP-kanMX-loxP cassette were then transformed with the plasmid containing Cre-recombinase to excise the sequence between two loxP sites. Cre-recombinase was induced by culturing the cells overnight in the YP-raffinose medium and then for 2-3 hr in YP-galactose medium. KanMX excision was confirmed by PCR. Dissection of tetrads was performed using a Singer MSM 300 microdissector (Delneri et al. 2003). Haploids displaying the BY4742 metabolic background were chosen after series of cultures on SD solid medium lacking Lys, Met, or Ura.

The effect of the loxP deletion was to replace the 204-bp (GLC7 ncRNA deletion mutant) and 140-bp (GLC7 control deletion mutant) intronic regions with $139 \mathrm{bp}$ of the loxP scar with the fragments of the transformation vector. We also constructed an insertion mutant with the 139-bp remnant of the cassette inserted into the middle of the predicted RNA structure without deleting any intron bases. All primers used in the creation of mutant strains are listed in File S2.

To perform the rescue experiment of the GLC7 ncRNA deletion mutant, the wild-type (WT) GLC7 ncRNA sequences were amplified and inserted in both sense and antisense orientations into a modified version of the pRS315 yeast shuttle vector (American Type Culture Collection, 77144), containing the constitutively expressed TDH3 promoter and LEU2 auxotrophic marker. The pRS315 TDH3 ncRNA recovery plasmids were transformed into BY4724 and the ncRNA deletion strain, following the method described in Gietz and Schiestl (2007). As a control, empty pRS315 was transformed into BY4742. Cells containing the plasmids were selected for by growth on SD -Leu media and successful PCR amplification of the plasmid.

\section{Growth rate assay}

Growth properties of the BY4742 strain, the intron mutants, and rescue mutants were assessed by time course growth profiles obtained using a FLUOstar optima microplate reader. Cells were cultured to stationary phase in YPD or F1 medium. The OD was measured at $595 \mathrm{~nm}$ and the cultures were diluted to an OD at $595 \mathrm{~nm}$ of 0.1 with prewarmed YPD media, F1 media, or F1 media containing $0.9 \mathrm{M} \mathrm{NaCl}$. Each of the 96-well plates was filled with $240 \mu \mathrm{l}$ of diluted culture or media control. Absorbance measurements were taken every 5 min immediately after $1 \mathrm{~min}$ shaking. Growth curves were plotted and analyzed using
$\mathrm{R}$ according to a modified version of the method specified previously (Norris et al. 2013). In brief, the area under each growth curve (AUC) was calculated by the pracma package from normalized absorbance data. Additionally, we fitted growth curves from data points taken every 30 min using grofit R package with default settings (Kahm et al. 2010). Maximum growth rate, lag phase, and maximum growth were calculated from the fitted curves.

\section{Competitive growth test}

Competitive growth assays were performed in $8 \mathrm{ml}$ media by adding an equal number of cells $\left(2 \times 10^{5}\right.$ cells $\left./ \mathrm{ml}\right)$ of a mutant strain and the BY4742 reference strain, which had the $H O$ gene replaced with kanMX as a marker to facilitate selection between strains. The GLC7/reference competition was performed in F1 media containing $0.9 \mathrm{M} \mathrm{NaCl}$. The cultures were grown at $30^{\circ}$ and maintained in log phase by diluting each culture to $2 \times 10^{5}$ cells $/ \mathrm{ml}$ in fresh media every $12-24 \mathrm{hr}$ until the generation number $37 \pm 1$ or $50 \pm 2$ was attained for $\mathrm{F} 1+0.9 \mathrm{M} \mathrm{NaCl}$ or YPD media, respectively. The number of generations was calculated as described by Parenteau et al. (2008). When the appropriate generation number had been reached, $\sim 200$ cells were plated on YPD media and after 2 days replicated onto YPD + geneticin. Cells were counted to obtain the ratio of mutant $v s$. reference strains.

\section{Data availability}

Strains are available upon request. Figure S1, Figure S2, Figure S3, Figure S4, Figure S5, and Figure S6 contain images of full gels used for creating Figure 2. Figure S7 contains additional analysis of growth of GLC7 mutants in F1 and F1 + $\mathrm{NaCl}$ media. Figure S8 contains negative result of the ncRNA rescue experiment. Table $S 1$ contains a list of fungal genomes used in the study. File S1 contains a list of RNAz, Cmfinder, and EvoFold predictions for each intron. File S2 contains primer sequences and probes used in the study. File S3 contains expression of introns and coding sequences of host genes (in RPKM) and the average percentile from exosome targets data (CRAC) for each gene. This article reanalyzed two publically available data sets by Schneider et al. (2012), GEO accession no. GSE40046, and by Hooks et al. (2014), GEO accession no. GSE58884.

\section{Results}

\section{Predictions of RNA structure within introns}

Since a predicted secondary structure of a single sequence is not generally sufficient to distinguish between a functional RNA and random sequences (Rivas and Eddy 2000), most RNA prediction methods require multiple homologous sequences. Thus our first step in RNA prediction was to identify orthologs of $S$. cerevisiae introns in other fungi. We searched for orthologs of intron-containing host genes in fully sequenced genomes and then for corresponding introns in those genes. In 36 fungal genomes, we were able to 
A
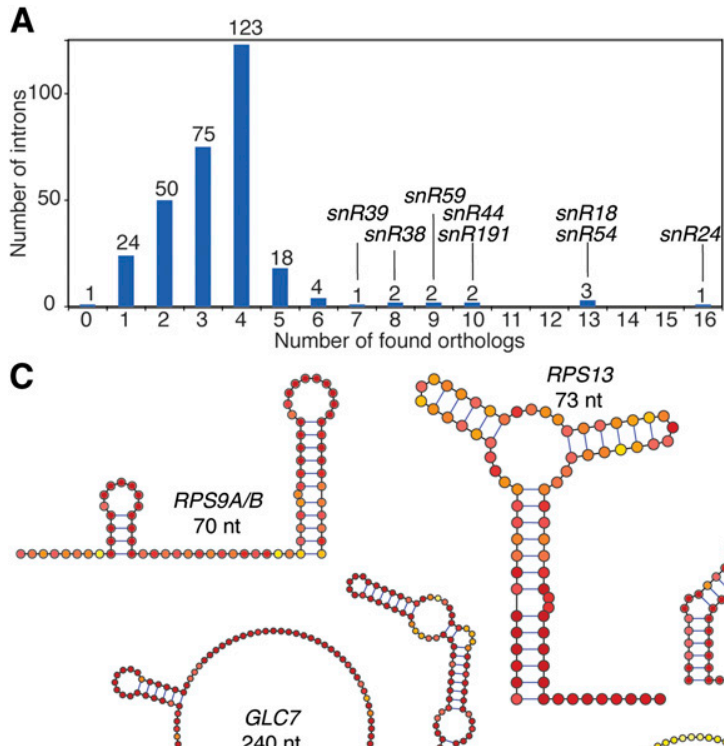

B
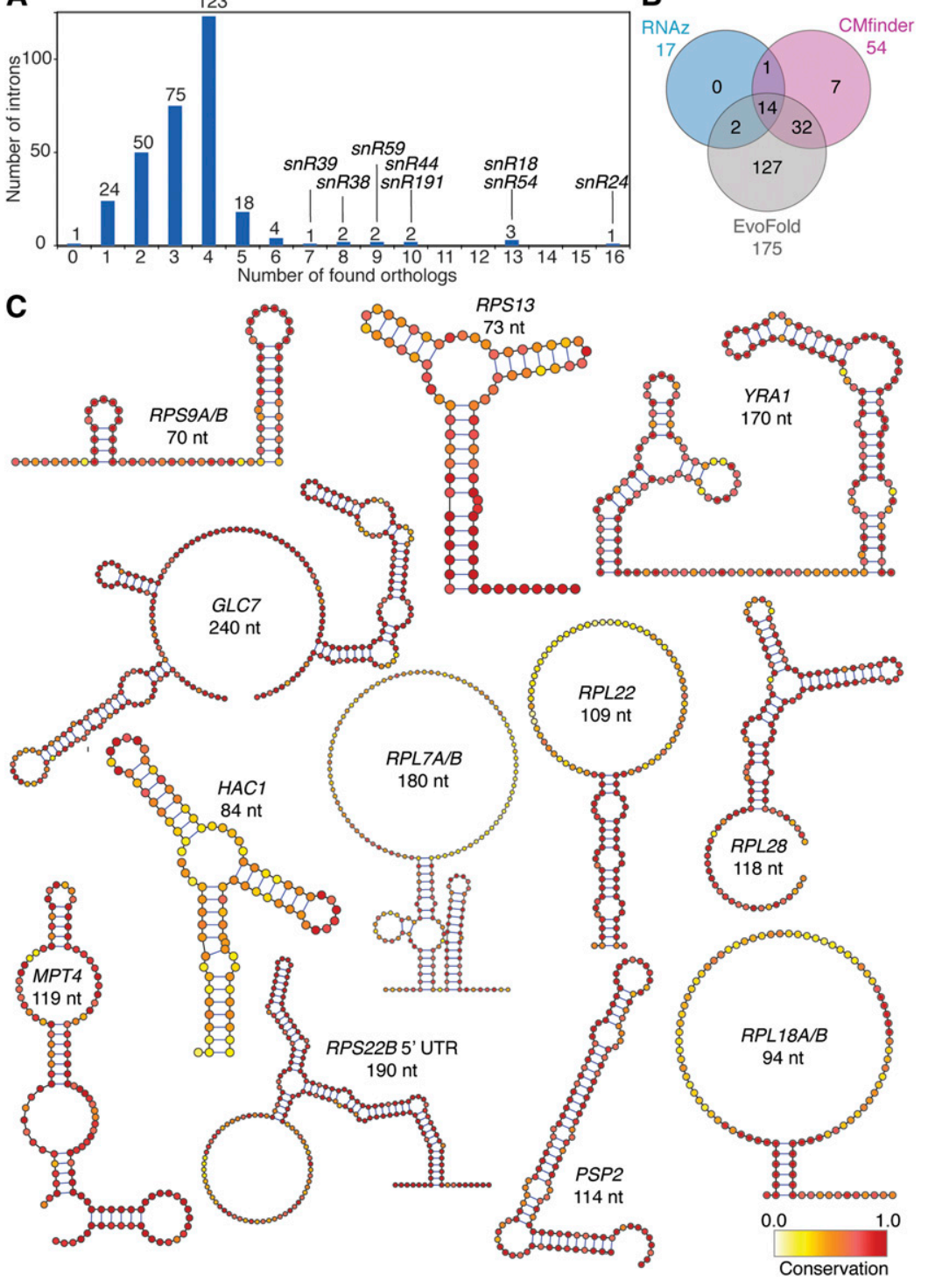

Figure 1 Predicted RNA structures. (A) For each intron of $S$. cerevisiae, the number of orthologous introns was counted among the 36 species included in the study. Plotted is the histogram of the number of introns ( $y$-axis) yielding a specific number of orthologs ( $x$-axis). Introns containing known snoRNAs are highlighted by name $(\mathrm{s} n \mathrm{R})$. (B) Venn diagram showing the number of common RNA structures predicted by RNAz, Cmfinder, and EvoFold. For example, there are 14 introns with structures predicted by all programs. (C) Predicted consensus RNA structures of selected introns. For each chosen intron, an iterative procedure of searching for orthologous sequences and extending the predicted RNA structure resulted in a multiple sequence alignment, which was collapsed to a consensus sequence with a secondary structure. For each structure, the gene name and the length of the predicted structured region are shown. In the case of duplicated ribosomal gene introns where both paralogous introns share a similar structure, both gene names are given. Structure images were prepared using VARNA (Darty et al. 2009). identify at least two orthologs for 281 introns and at least one ortholog for 305 introns (Figure 1A). Only the intron of YDR535C does not have an ortholog in any of the species searched, but annotation of this sequence as a gene is dubious. The vast majority of introns are conserved only in the Saccharomyces sensu stricto, and we identify fewer than four orthologs for each intron on average. In contrast, orthologs of yeast introns containing known intronic snoRNAs are found in a wider range of fungal genomes, having on average 9.6 orthologs (Figure 1A).

Orthologous intronic sequences were used to predict novel RNA structures using three independent computational methods: CMfinder, RNAz, and EvoFold. Each of these approaches yielded a very different number of predicted RNA structures in introns. RNAz, CMfinder, and EvoFold identified putative conserved RNA structures in 17, 54, and 175 introns, respectively. We found 14 structures in the intersection of all three approaches, within the only intron of each of GLC7, HAC1, IMD4, MPT5, RPL18A, RPL18B, RPL22B, RPL28, RPS9A, RPS9B, and RPS13, in the first intron of $R P L 7 A$, and in both introns of RPS22B (Figure 1B, File S1).

For further bioinformatic and experimental analysis, we decided to focus on the introns that had structures predicted by all three programs, together with their paralogs (i.e., introns in RPL22A and RPL7B), and three introns with high prediction scores in at least two of the three approaches (i.e., introns in NOG2, YRA1, and PSP2). We used the INFERNAL software (Nawrocki et al. 2009) and the Rfam library of covariance models to search the intron sequences for known ncRNA classes (Gardner et al. 2010). Besides the known snoRNAs, our other high-scoring RNA predictions do not resemble any previously known RNA families. We also used an iterative procedure combining covariance model searches using the INFERNAL package and manual inspection of multiple sequence alignments to identify additional homologs of 
our predicted structures in more distant species (Table 1, Figure 1C). We find that the known snoRNAs are very well conserved among Fungi: snR191 orthologs were found in all Saccharomycotina, snR44 in Saccharomycotina and Pezizomycotina, while snR54 is present in some Metazoan genomes as well as Fungi. The previously known HAC1 intron-exon structure is also conserved in Fungi and Metazoa (Hooks and Griffiths-Jones 2011). Iterative INFERNAL searches for homologs of the predicted structures in the introns of RPL18, RPL22, RPL7, and RPS9 allowed us to extend the conservation to the Saccharomyces and Candida clades. The identification of highly similar short motifs in the alignments of RPL7, RPL18, RPL22, RPL28, and RPS9 introns demonstrated that predicted structures within introns are well conserved in Saccharomyces sensu stricto. The high sequence conservation means that relatively few compensatory mutations support these conserved predicted structures. Only in the case of the intron of RPS13 is there evidence for maintenance of the RNA structure through multiple compensatory mutations.

\section{Experimental detection of the predicted intronic ncRNA}

In order to determine whether the predicted intronic RNAs are expressed and maintained in the cell, we performed RT-PCR on total and low molecular weight cDNA from haploid BY4741 WT $S$. cerevisiae strain. We designed three sets of specific primers to amplify the fragment of the intron with the predicted structure or snoRNA, the entire intron, and part of the exons flanking the intron. As a positive control for the PCR, we used the genomic DNA to amplify products with the primer pairs described above in the snR44, snR191, and snR54 snoRNA genes. Negative controls for all PCR experiments were performed with no template added to the reactions.

If a bona fide ncRNA product is processed from an intron, we expected to obtain bands corresponding to the spliced mRNA of the host gene, and also for the region of the predicted structure, but not the larger product from the complete intron. This pattern was observed for the RPS $22 B$ intron harboring the known snoRNA snR44 and for two other introns with predicted structures, namely, the intron of GLC7 and the first intron of $R P L 7 B$, thus confirming these sequences as novel ncRNAs (Figure 2A, Figure S1). A similar pattern was also observed for MPT5, although its expression was very low (Figure 2A, Figure S1C). The RT-PCR corresponding to snR191 in NOG2 displayed a pattern indicative of complete splicing of the host mRNA, but also showed the maintenance of the complete intron and the predicted ncRNA. We refer to these sequences as "introns maintained" in the cell after splicing as opposed to "introns retained" in the pre-mRNA. We observed a similar pattern of correct splicing with intact intron and ncRNA maintenance for our predicted structures in the introns of RPS13, RPS9B, RPL7A, $R P L 22 A$, and in the $5^{\prime}$ UTR intron of $R P S 22 B$ (Figure 2B, Figure S2, Figure S3). These data show that a mixed population of mature ncRNAs and spliced but unprocessed introns
Table 1 Conservation of RNA structure predictions

\begin{tabular}{|c|c|c|c|}
\hline No. & Locus & Gene & Conservation \\
\hline 1 & $\begin{array}{l}\text { YBR189W } \\
\text { YPL081W }\end{array}$ & $\begin{array}{l}R P S 9 B \\
R P S 9 A\end{array}$ & $\begin{array}{l}\text { Saccharomycetaceae and } \\
\text { Candida sp. }\end{array}$ \\
\hline 2 & YDR064W & RPS13 & Saccharomycetaceae \\
\hline 3 & YDR381W & YRA1 & Saccharomycetaceae \\
\hline 4 & YER133W & GLC7 & Saccharomyces sensu stricto \\
\hline 5 & YFL031W & HAC1 & Fungi and Metazoa \\
\hline 6 & $\begin{array}{l}\text { YFL034C-A } \\
\text { YLR061W }\end{array}$ & $\begin{array}{l}\text { RPL22B } \\
\text { RPL22A }\end{array}$ & Saccharomycetaceae \\
\hline 7 & $\begin{array}{l}\text { YGL076C } \\
\text { YPL198W }\end{array}$ & $\begin{array}{l}\text { RPL7A } \\
\text { RPL7B }\end{array}$ & $\begin{array}{l}\text { Saccharomycetaceae and } \\
\text { Candida sp. }\end{array}$ \\
\hline 8 & YGL103W & RPL28 & Saccharomyces sensu stricto \\
\hline 9 & YGL178W & MPT5 & Saccharomyces sensu stricto \\
\hline 10 & $\begin{array}{l}\text { YLR367W } \\
\text { 5' UTR }\end{array}$ & RPS22B & $\begin{array}{l}\text { Saccharomycetaceae except } \\
\text { L. lactis }\end{array}$ \\
\hline 11 & $\begin{array}{l}\text { YLR367W } \\
\text { snR44 }\end{array}$ & $R P S 22 B$ & $\begin{array}{l}\text { Saccharomycotina and } \\
\text { Pezizomycotina }\end{array}$ \\
\hline 12 & YML017W & PSP2 & Saccharomyces sensu stricto \\
\hline 13 & $\begin{array}{l}\text { YML056C } \\
\text { SnR54 }\end{array}$ & IMD4 & Saccharomycetales and Diptera \\
\hline 14 & $\begin{array}{l}\text { YNL301C } \\
\text { YOL120C }\end{array}$ & $\begin{array}{l}\text { RPL18B } \\
\text { RPL18A }\end{array}$ & $\begin{array}{l}\text { Saccharomycetaceae and } \\
\text { Candida sp. }\end{array}$ \\
\hline 15 & $\begin{array}{l}\text { YNR053C } \\
\text { SnR191 }\end{array}$ & NOG2 & $\begin{array}{l}\text { Saccharomycetaceae and } \\
\text { Candida sp. }\end{array}$ \\
\hline
\end{tabular}

are present in the cell. We also found that the mRNA transcripts of IMD4 (containing snR54 in its intron), PSP2, RPS9A, RPL28, RPL22B, RPL18A, RPL18B, and YRA1 were present in both spliced and unspliced forms, supporting the existing evidence that intron retention is the most common case of alternative splicing in yeast (Plass et al. 2012) (Figure $2 \mathrm{C}$, Figure S4, Figure S5). We found no evidence of splicing of HAC1 under the specific experimental conditions we tested (see Figure S6A). As negative controls, we conducted the same RT-PCR analysis on six intron-containing genes that had no predicted RNA structures. None of the six displayed patterns consistent with ncRNAs processed from the introns. The genes YBR219C and BMH2 did not appear to be expressed and the four ribosomal protein genes RPL27A, RPS27B, $R P S 16 A$, and RPS19B showed the intron-maintained pattern (Figure S6, B-G). RNA-seq data show higher expression of introns in ribosomal protein genes that contain predicted RNA structures.

We also validated the presence of expressed intronic sequences in the cell by reanalyzing deep sequencing data of total RNA extracted from $S$. cerevisiae (Hooks et al. 2014). We counted all reads overlapping introns and normalized by the intron length and the total number of reads mapped to obtain RPKM values. Median intron expression was 15.3 RPKM. We observed that 17 of 19 introns with predicted structures have evidence of expression (more than 150 reads or 20 RPKM), seven of which fell within the 90th percentile of intron expression.

Since a third of all introns are found in highly expressed ribosomal protein (RP) genes, we next measured the relative expression levels of maintained introns in RP that contain or do not contain predicted structures. Since the mRNA levels of 

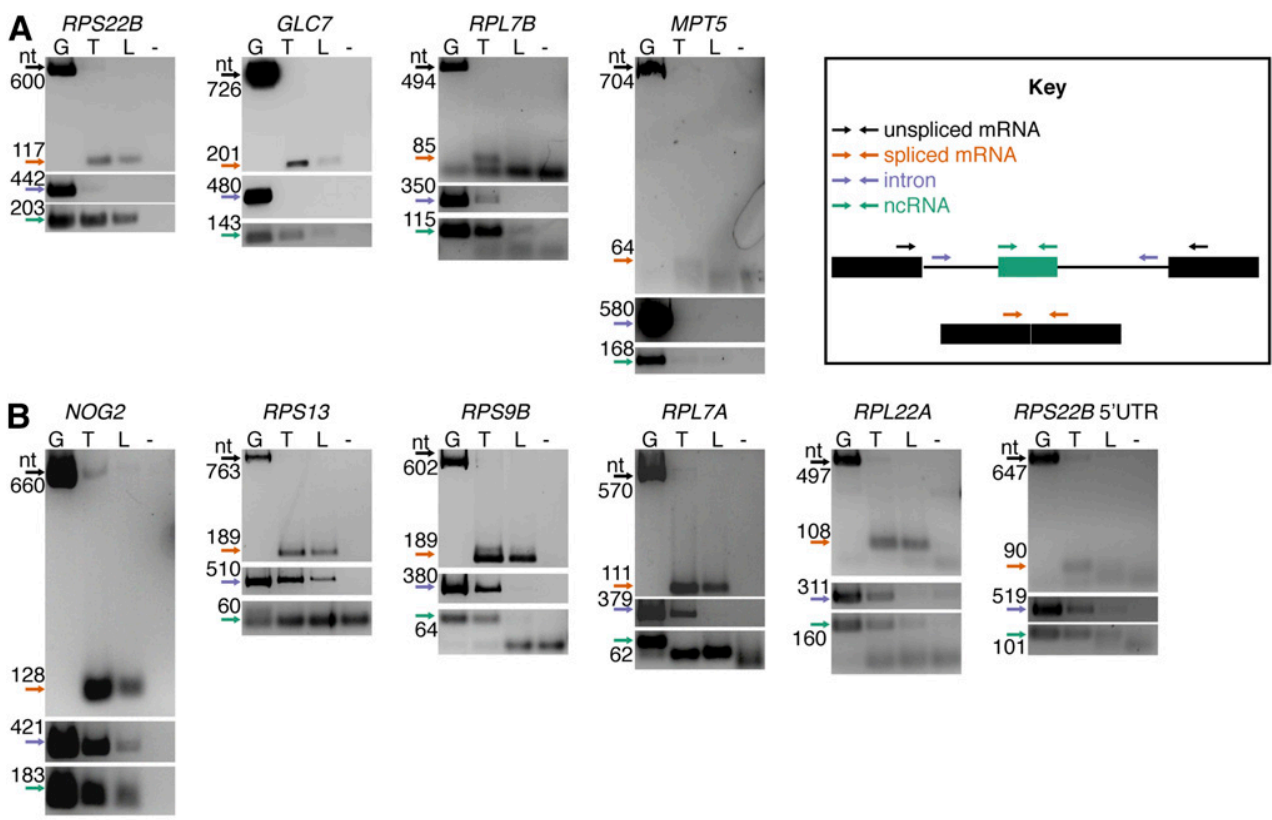

C ${ }_{\text {IMD4 }}$
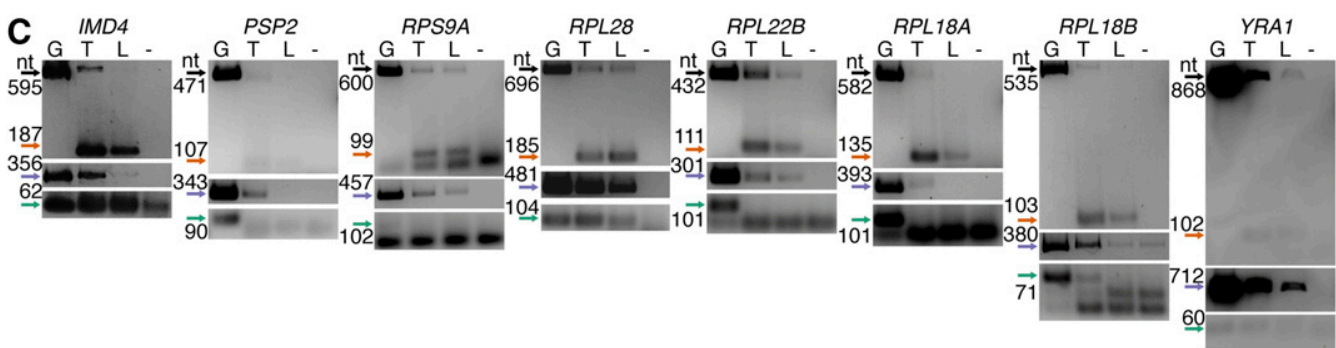

Figure 2 RT-PCR confirmation of intron fates conducted on total RNA and low-weight-enriched RNA using random priming. PCR of genomic DNA was used as positive control. (A) Agarose gel confirming ncRNAs expressed from introns and maintained in the cell. (B) Agarose gel confirming ncRNA expression accompanied by complete mRNA splicing. (C) Agarose gel confirming ncRNA expression accompanied by alternative splicing. Arrows indicate the expected size of the PCR products according to the key. Lane designations for DNA templates: G, Genomic DNA (positive control); $\mathrm{T}$, total CDNA; L, low-molecularweight-enriched cDNA; and -, no template negative control. With the exception of GLC7 ncRNA, which was run on a separate gel, the images of mRNAs, introns, and ncRNAs for each gene were cropped from the same agarose gel picture with brightness and contrast applied equally across the entire image (full images available in Figure S1, Figure S2, Figure S3, Figure S4, Figure S5, and Figure S6). For small-size PCR products, cropping included primer dimers. the genes hosting predicted intronic structures appeared to be greater than the average mRNA amount for the entire set of intron-containing genes, we also normalized the intron expression levels by the level of their host gene transcript. Interestingly, expression of RP introns with predictions was significantly higher compared with all RP introns before and after normalizing for host gene expression (median RPKM 65.5 compared to 33.0; Mann-Whitney $U$-test $P$-value $=$ 0.006 and median normalized expression 0.11 compared to 0.03; Mann-Whitney $U$-test $P$-value $=0.014$ ) (Figure $3 \mathrm{~A})$.

\section{Introns with predicted RNA structures are more likely to be targeted by the exosome}

We hypothesize that introns with RNA structures either contain novel ncRNAs or are involved in the regulation of premRNA splicing; in both cases, the host transcript would be expected to associate with the exosome complex. We analyzed the data of Schneider et al. (2012), who used in vivo RNA cross-linking (CRAC) of exosome components to show that noncoding RNAs, snoRNAs, pre-tRNAs, and pre-mRNAs are the most prominent exosome targets. The Schneider et al. (2012) data set (GEO accession no. GSE40046) contains deep sequencing reads from 16 separate in vivo cross-linking experiments to the tagged protein components of the exo- some. When we normalized the number of reads of each intron-containing gene from GSE40046 by the average number of reads for this intron-containing gene from GSE58884, we observed that the set of genes with both known snoRNA host genes and our novel RNA predictions appears to be targeted by the exosome machinery more than expected (our predictions, Mann-Whitney $U$-test, $P=2.44 \times 10^{-4}$; snoRNAs, $P=$ 0.005) (Figure 3B). This suggests that the introns of interest are either retained in pre-mRNAs or contain noncoding RNAs. Taken in conjunction with our RT-PCR data, the CRAC data indicated that the pre-mRNA transcripts of PSP2, RPS9A, $R P L 18 A$, and $R P L 18 B$ are maintained in the cell, possibly due to the stable secondary structures that are resistant to the degradation by exosome. In contrast, the presence of a novel ncRNA was indicated for the MTP5 intron.

\section{Function of the GCL7 intronic nCRNA}

The intronic sequence in GLC7 was recently shown to play a role in the cellular response to osmotic pressure (Parenteau et al. 2008). We therefore investigated further the putative ncRNA derived from this intron. First, we used Northern hybridization to confirm the RT-PCR analysis of expression of the ncRNA and confirm the strand from which it is expressed, since the computational predictions indicated that the stable 


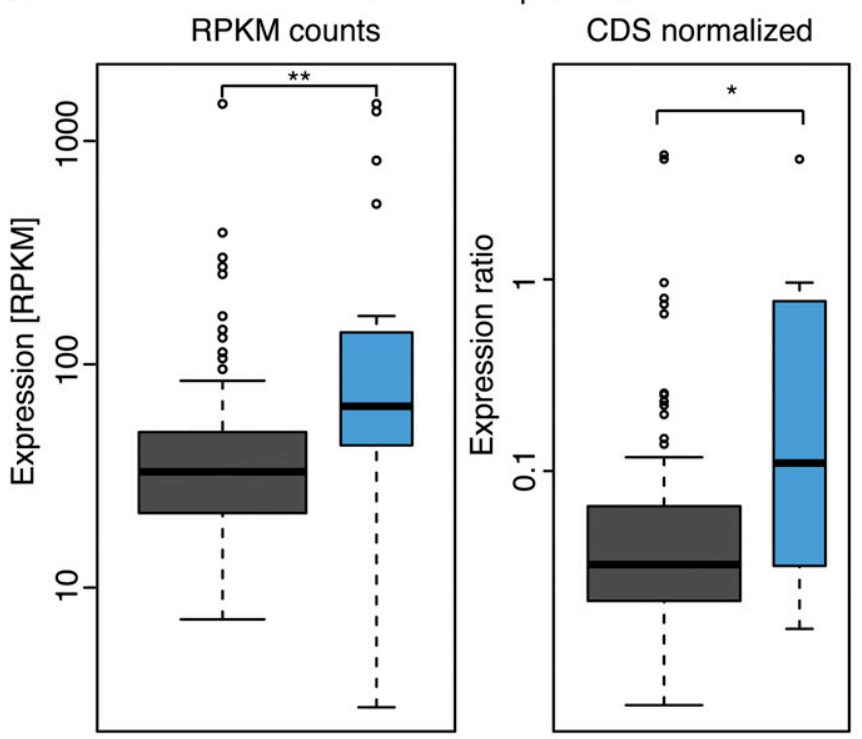

B

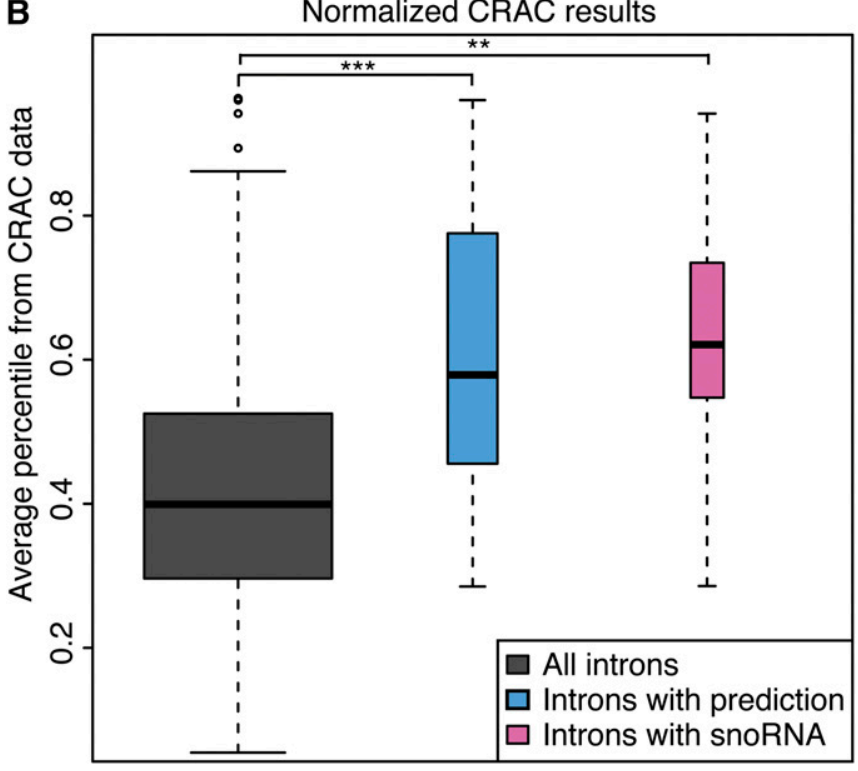

Figure 3 Properties of loci containing intronic novel RNA predictions or snoRNAs. Quantifications were based on RNA-seq data of the same total RNA as used in Figure 2 (GEO accession no. GSE58884) and on CRAC data (GEO accession no. GSE40046). (A) Box plots showing ribosomal protein intron transcript levels in RPKM without normalization and with normalization to host gene transcript levels. Values are shown for all RP gene introns and 12 RP gene introns with predictions. (B) Association of introns with the exosome. We reanalyzed 16 independent sequencing experiments by Schneider et al. (2012) of RNA fragments cross-linked to exosome components. Reads mapping to each intron were normalized by the host gene expression estimated by RNA-seq. For each CRAC experiment an intron was given a percentile value of how frequently it was bound to an exosome component compared to other introns. Values presented are averaged percentile derived from 16 experiments and are shown for all introns, the 19 introns with predictions, and the eight introns containing snoRNAs. The levels of significance for the Mann-Whitney $U$-tests are represented as follows: *** $P<10^{-3} ; * * P<0.01 ; * P<0.05$
RNA structure can be formed by this GLC7 intron region in both orientations. Strand-specific probes suggest expression of a 250-nt molecule from the antisense strand of the GLC7 intron (Figure 4A, left panel). Second, we employed primer walking to define the $5^{\prime}$ and $3^{\prime}$ ends of the ncRNA (Figure 4B). We were able to specifically define GLC7 ncRNA boundaries, which are different from those of the previously annotated CUT568, which is also antisense to the GLC7 gene (Figure 4C). The sizes and positions of PCR products, Northern probes used, and computational predictions are shown in Figure 4C.

To study the phenotype of the ncRNA, we used two approaches: deletion and alteration of the ncRNA structure, the latter of which increases the distance between the two predicted hairpins. We deleted two regions in the intron of GLC7 via PCR-mediated gene deletion and the cre-loxP system: an intronic region overlapping with CUT568 but not with the ncRNA (negative control deletion mutant) and the intronic region corresponding to the ncRNA (GLC7 ncRNA deletion mutant). For the alteration, we inserted $139 \mathrm{bp}$ in the middle of the predicted ncRNA to modify its structure (GLC7 ncRNA insertion mutant; see Materials and Methods). Previously it was shown that replacing the GLC7 gene with its cDNA decreases the cell viability in $\mathrm{NaCl}$ stress (Juneau et al. 2006; Parenteau et al. 2008). In order to determine whether this defect was due to the ncRNA structure rather than the intron in itself or the CUT568, we tested all engineered strains with the mutated GLC7 intron in F1 medium and in F1 medium containing $0.9 \mathrm{M} \mathrm{NaCl}$ (Figure 5A). In F1 supplemented with $0.9 \mathrm{M} \mathrm{NaCl}$, we observed a significant difference in growth, as estimated by the AUC (Norris et al. 2013), for both GLC7 ncRNA deletion and insertion mutants compared with the WT (one-way ANOVA with Dunnett's multiple comparison test: for both mutants $P<0.0001$ ) or the negative control deletion (for both mutants $P<0.0001$; Figure 5B). Specifically, we found that in the $\mathrm{F} 1+0.9 \mathrm{M} \mathrm{NaCl}$ media, the lag phase $(\lambda)$ is longer in the GLC7 ncRNA deletion and insertion mutants. Moreover, their maximum growth rate $(\mu)$ and the final biomass after $48 \mathrm{hr}$ (A) are decreased, whereas for the negative control deletion mutant those parameters are the same as the WT (Figure S7). We attempted to restore the fitness of the GLC7 ncRNA deletion mutant by inserting the ncRNA expressed under the strong constitutive promoter (see Materials and Methods). There was no significant recovery in fitness from the addition of the sense or antisense orientation ncRNA overexpression plasmids (Figure S8). This suggests that the intronic ncRNA is exclusively cis-acting.

We further verified the phenotypic difference of the mutant using one-to-one competition experiments. The WT BY4742 strain and the GLC7 deletion mutant were grown together in F1 medium supplemented with $0.9 \mathrm{M} \mathrm{NaCl}$, and the composition of the population was analyzed after 37 generations. A significant drop in relative amount of the mutant strains was detected with just over $25 \%$ of total cells being GLC7 deletion mutant, instead of the expected $50 \%$ (Student's $t$-test, $P=$ $7.30 \times 10^{-5}$ ). Taken together, these data further indicate that the deletion of the putative ncRNA in the GLC7 intron 
A

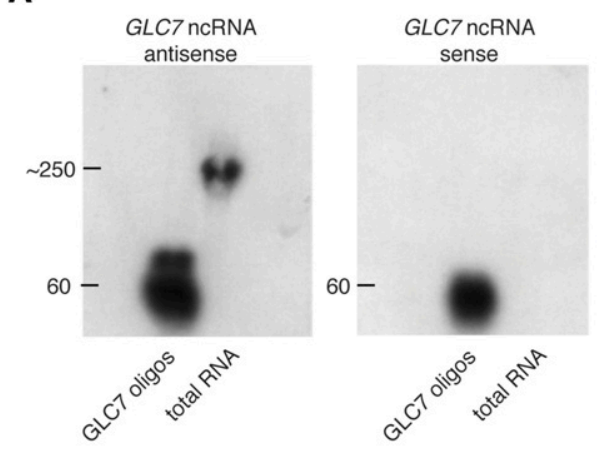

C

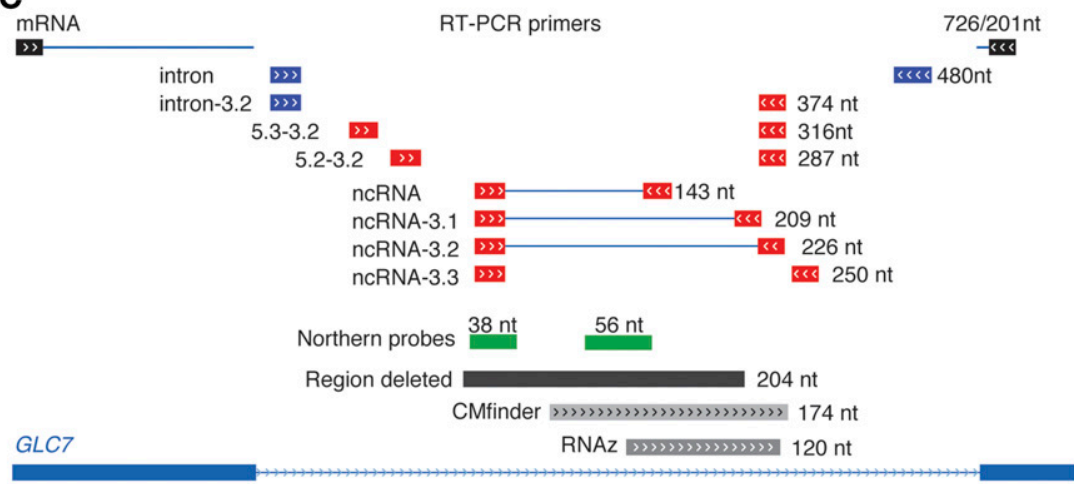

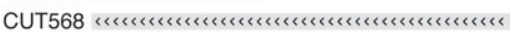

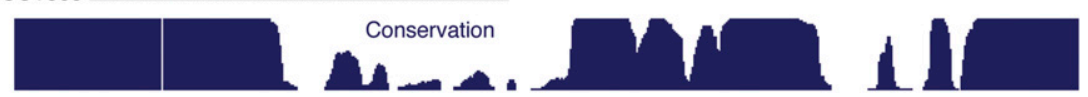

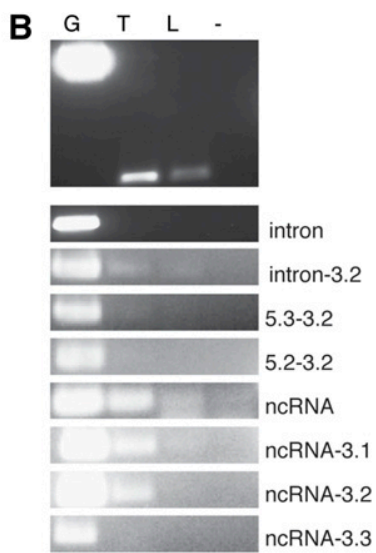

Figure 4 Characterization of the size and expression of the nCRNA within GLC7 intron. (A) Northern blot of $S$. cerevisiae total RNA and oligos mimicking GLC7 intron. The blots were probed with strand-specific probes showing expression of ncRNA in antisense (left panel) and sense orientation (right panel) to the GLC7 gene. The estimation of the sizes is based on the 60-nt oligos visible on the film and a comparison with the markers visible on the membrane. (B) RT-PCR on total RNA and low-weight-enriched RNA using random priming showing the expression of ncRNA within the GLC7 intron. PCR of genomic DNA was used as positive control. Names of the primers are listed on the right and are the same as indicated in $C$. Gel annotation: G, genomic DNA; T, total CDNA; L, lowmolecular-weight-enriched cDNA; and - , no template negative control. (C) Data uploaded into the University of California Santa Cruz genome browser for sequence annotation and data visualization presenting annotated GLC7 intron. Primer names used for RT-PCR are listed next to black, blue, and red boxes indicating their position with respect to the gene annotation below and the size of the corresponding PCR product. Lines joining primers mark the amplified sequences; the regions targeted for deletion by the loxP method are symbolized by the black box; the location of the strand-specific Northern probes are shown in green; and regions with the putative structure predicted by RNAz and CMfinder are shown in gray. All features map directly onto the fragment of the gene structure diagrams. The bottom panel represents the degree of conservation of gene regions among seven yeast species. is responsible for the observed impairment of cell growth rate and competitive fitness during salt stress, whereas deletion of the upstream intronic region overlapping the CUT568 expressed from the opposite strand has no impact on the phenotype in salt stress.

We then looked at the expression of GLC7 via real-time PCR in WT, control deletion mutant, and GLC7 ncRNA deletion mutant. The data show that the deletion of the ncRNA or the control region in GLC7 intron does not impair GLC7 expression in F1 media. In fact, the GLC7 mRNA level in ncRNA deletion mutant appears to be slightly elevated compared to that of WT or control deletion (mean \pm SEM: $1.20 \pm$ 0.01). However, in salt stress the GLC7 mRNA level of the GLC7 ncRNA deletion mutant is reduced to $0.57 \pm 0.03$ of that of the WT (Figure 5C), whereas the GLC7 mRNA level in control deletion decreases only by a small amount (mean \pm SEM: $0.87 \pm 0.02$ ). We suggest that the phenotypic effect of the ncRNA knockout under salt stress may be due to the decreased expression or a splicing defect of GLC7 in the GLC7 ncRNA deletion mutant.

\section{Discussion}

ncRNAs can be present in intergenic regions (David et al. 2006), inside exons, as in the case of ncRNA derived from TRM10 mRNA (Pircher et al. 2014), or within introns (Ooi et al. 1998; Nakaya et al. 2007). Using multiple computational methods, we predicted stable and conserved RNA structures in 19 introns, 12 of which are present in RP genes. By RT-PCR, we validated the presence of the predicted ncRNAs and, in several cases, of the whole introns. Our predicted RNA structures include in the $5^{\prime}$ UTR intron of RPS22B (Figure 2B) and in the intron of RPL18A (Figure 2C). Both introns have been previously reported to trigger RNase IIImediated mRNA degradation by Rnt1p (Danin-Kreiselman et al. 2003). We also predicted and validated the expression of putative ncRNAs in the introns of RPS9A and RPS9B (Figure $2, B$ and $C$ ), and these introns were previously shown to regulate expression of both their host genes and their paralogs (Plocik and Guthrie 2012). In another study on the effects of intron deletion (Parenteau et al. 2011), 11 RP introns besides RPS13 were shown to regulate the expression of the host gene or its paralogous copy, change cell sensitivity to drugs, or alter the competitive fitness when deleted. Our RNA-seq data analysis shows that introns in RP genes containing RNA structures are significantly more expressed than the introns in RP genes lacking a predicted ncRNA. Among the seven non-RP proteins with high-scoring predictions, only the PSP2 introns have no previously suggested function. HAC1 and YRA1 introns contain structures that regulate their own splicing and IMD4 and NOG2 contain snoRNAs that guide chemical modification of other RNAs, whereas GLC7 


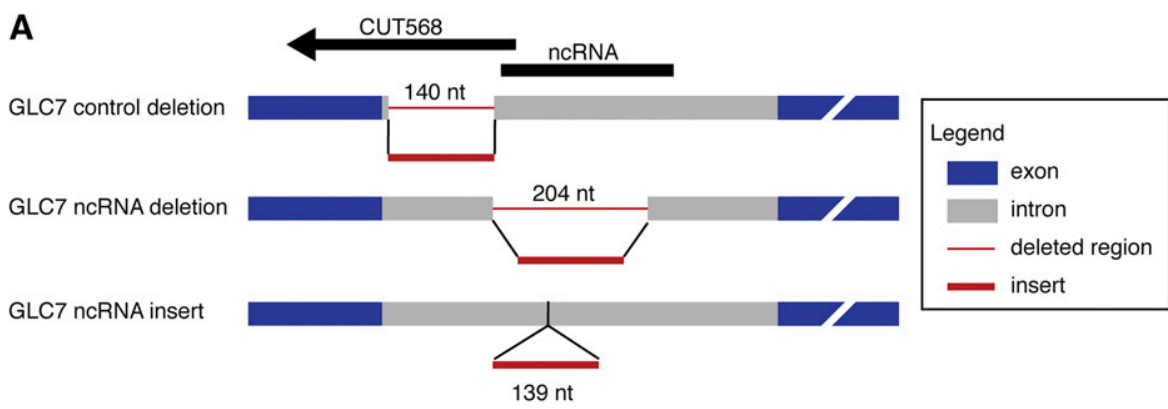

Figure 5 Effects of GLC7 intron mutation. (A) Schematic representation of intron mutants used for phenotype studies. (B) The GLC7 ncRNA deletion and GLC7 ncRNA insertion mutants disrupting the structure sequence of the GLC7 intron were compared with the WT strain and the intronic negative control in $\mathrm{F} 1$ medium containing $0.9 \mathrm{M} \mathrm{NaCl}$. Values in the box plots

B

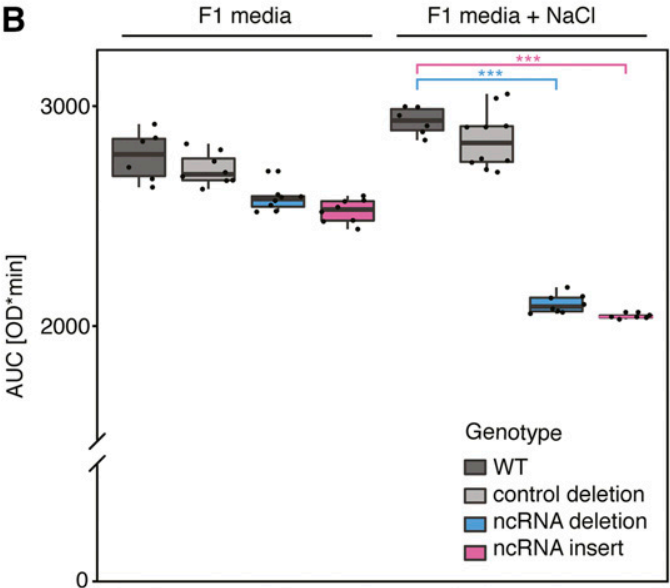

C

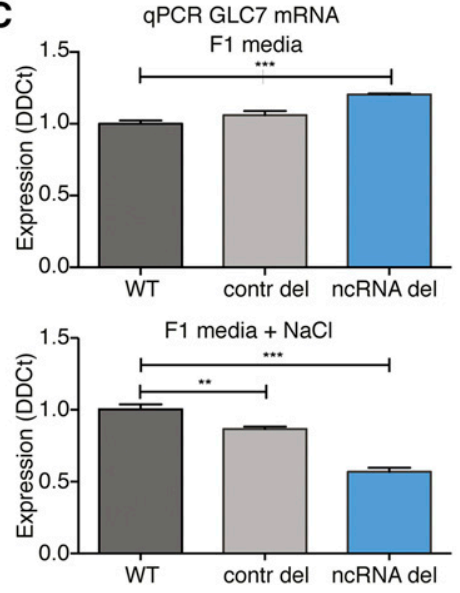
present the means of the AUC as determined by the $R$ pracma package. Significance estimated by one-way ANOVA with Dunnett's multiple comparison test $(* * * P<0.0001)$. (C) Expression levels (average expression with SEM) of GLC7 mRNA in the WT, control mutant, and the GLC7 ncRNA deletion mutant grown in $\mathrm{F} 1$ and $\mathrm{F} 1+0.9 \mathrm{M} \mathrm{NaCl}$ media, assessed by RT-qPCR. Significance estimated by one-way ANOVA with Dunnett's multiple comparison test $(* * * P<0.0001, * * P=$ 0.005).

and MPT5 introns appear to be required for stress tolerance (Parenteau et al. 2008). Our data raise the possibility that, at least in some cases, an intronic RNA structure is responsible for the biological function, rather than the intron itself.

Our experimental characterization and follow-up analysis have allowed us to generate hypotheses regarding the mechanism of function of specific sequences. For example, the GLC7 intron was previously shown to mediate the response to salt stress (Parenteau et al. 2008) and we now demonstrate that the factor responsible for the biological function is an intronic sequence with a discreet RNA structure (Figure 5). Furthermore, characterization of the stable ncRNA by RT-PCR and Northern combined with unsuccessful rescue experiment suggests that the predicted structure functions in cis. RNAs functioning in cis are common among eukaryotes and originate from their own transcriptional unit (Quinn and Chang 2016). There is a prevalence of antisense ncRNA transcripts across the yeast (Neil et al. 2009) and mammalian genomes (Core et al. 2008) and such ncRNAs are able to regulate the expression of the gene on the opposite strand in a variety of ways including: transcriptional interference (Houseley et al. 2008; Hainer et al. 2011), alternative splicing (Wang et al. 2005), and at the translational level (Carrieri et al. 2012).

New techniques, such as in vivo RNA cross-linking to protein complexes coupled with next-generation sequencing, can aid the discovery of novel pathways involving all types of ncRNA, including those encoded in introns. Our analysis of exosome target data presented by Schneider et al. (2012) indicates genes with intronic RNA structure predictions are more likely to be transcriptionally regulated or contain novel ncRNAs.
With the exception of a few snoRNAs, $S$. cerevisiae introns do not appear to contain classical intronic ncRNAs. Although the function of most intronic RNAs in higher eukaryotes is still unknown, the evidence of tissue-specific expression (Louro et al. 2008) and binding to protein complexes known to promote epigenetic modifications (Guil et al. 2012) indicates that intronic transcripts may have specific functions, rather than arising from spurious transcription or slow premRNA turn-over. Our results provide clear evidence for the function of the GLC7 intronic RNA structure in an intron-poor single-celled fungal species.

Our work, and that of others mentioned here, raises interesting questions about the general nature of intron stability postsplicing. Introns removed by splicing have been thought to be rapidly degraded and the main focus of intron biology has concerned elucidation of splice sites and the arrangement of the splicing machinery. For example, it was observed that the deletion of the debranching enzyme promotes the accumulation of lariat introns (Chapman and Boeke 1991), so it was assumed that all introns are rapidly debranched and targeted for degradation in normal cells. Tiling arrays and next generation sequencing have readily shown that some intronic sequences are abundant in the cell, but they are usually dismissed as remnants of normal splicing or part of immature pre-mRNAs (Louro et al. 2009). Recently, the fate of introns themselves has been systematically assessed in Xenopus tropicalis embryos showing that $90 \%$ of introns are maintained in the nucleus postsplicing (Gardner et al. 2012) and $\sim 5 \%$ of genes generate lariats that are stable in the cytoplasm (Talhouarne and Gall 2014). Linear intron-derived 
ncRNAs with some similarities to snoRNAs have been found in HeLa cells and human embryonic stem cells (Yin et al. 2012). Most importantly, circular intronic RNAs increase the expression of their host genes as exemplified by gene ANKRD52 (Zhang et al. 2013). We and others observe that many of the introns examined are retained in the premRNA or maintained in the cell after splicing. This is consistent with the observations of Coleclough and Wood (1984) who were the first to described discrete intron products processed from the mouse immunoglobin pre-mRNA. Immunoglobulin mRNAs are expressed at high levels, like the ribosomal genes tested in our study. We therefore speculate that postsplicing intron stability might be a prevalent phenomenon for highly expressed genes and that intron products may be regulators of these highly expressed gene products.

\section{Conclusions}

We undertook a systematic approach using the well-studied S. cerevisiae genome in order to identify introns with undiscovered function. Comparing intron sequences of related yeast species, we found at least 19 introns contain putative conserved RNA structures. By RNA-seq and RT-PCR, we show that several of the intronic sequences containing secondary structures are not degraded after removal from pre-mRNAs. Furthermore, we show that RNA structures embedded in introns can be directly responsible for regulating gene expression and maintaining phenotype in the intron-poor yeast. For example, a small portion of the GLC7 intronic sequence, representing a novel RNA structure, plays an important role in the cellular response to salt stress. More generally, the cellular abundance of intron sequences from RP genes with predicted intronic RNAs is significantly higher than for those lacking such predictions. Overall, our data support the possibility that the presence of functional RNA structures in introns has contributed to selective intron retention in the Saccharomycetes.

\section{Acknowledgments}

We are grateful to James Allen for assistance with EvoFold predictions. K.B.H. was supported by a Wellcome Trust $\mathrm{PhD}$ studentship (grant WT086809). S.P. is supported by a Wellcome Trust Biomedical Resource and Biotechnology Development Grant (104981/Z/14/Z).

Author contributions: D.D. and S.G.-J. conceived the study; K.B.H., S.G.-J., and D.D. designed the experiments; K.B.H. performed the predictions, RT-PCRs, and RNA-seq analysis and constructed the GLC7 ncRNA deletion mutant; S.N. performed the real-time PCR and Northern blots, constructed the GLC7 negative control mutant, and characterized the growth of all GLC7 mutant strains; S.P. constructed ncRNA rescue mutants and characterized their fitness; and K.B.H., S.G.-J., and D.D. wrote the manuscript with input from S.N. and S.P.

\section{Literature Cited}

Baganz, F., A. Hayes, R. Farquhar, P. R. Butler, D. C. Gardner et al., 1998 Quantitative analysis of yeast gene function using competition experiments in continuous culture. Yeast 14: 1417-1427.

Carrieri, C., L. Cimatti, M. Biagioli, A. Beugnet, S. Zucchelli et al., 2012 Long non-coding antisense RNA controls Uchl1 translation through an embedded SINEB2 repeat. Nature 491: 454 457.

Carter, Z., and D. Delneri, 2010 New generation of loxP-mutated deletion cassettes for the genetic manipulation of yeast natural isolates. Yeast 27: 765-775.

Catalanotto, C., G. Azzalin, G. Macino, and C. Cogoni, 2002 Involvement of small RNAs and role of the qde genes in the gene silencing pathway in Neurospora. Genes Dev. 16: 790-795.

Chapman, K. B., and J. D. Boeke, 1991 Isolation and characterization of the gene encoding yeast debranching enzyme. Cell 65: 483-492.

Cheng, J., P. Kapranov, J. Drenkow, S. Dike, S. Brubaker et al., 2005 Transcriptional maps of 10 human chromosomes at 5-nucleotide resolution. Science 308: 1149-1154.

Chorev, M., and L. Carmel, 2013 Computational identification of functional introns: high positional conservation of introns that harbor RNA genes. Nucleic Acids Res. 41: 5604-5613.

Coleclough, C., and D. Wood, 1984 Introns excised from immunoglobulin pre-mRNAs exist as discrete species. Mol. Cell. Biol. 4: 2017-2022.

Core, L. J., J. J. Waterfall, and J. T. Lis, 2008 Nascent RNA sequencing reveals widespread pausing and divergent initiation at human promoters. Science 322: 1845-1848.

Danin-Kreiselman, M., C. Y. Lee, and G. Chanfreau, 2003 RNAse III-mediated degradation of unspliced pre-mRNAs and lariat introns. Mol. Cell 11: 1279-1289.

Darty, K., A. Denise, and Y. Ponty, 2009 VARNA: interactive drawing and editing of the RNA secondary structure. Bioinformatics 25: 1974-1975.

David, L., W. Huber, M. Granovskaia, J. Toedling, C. J. Palm et al., 2006 A high-resolution map of transcription in the yeast genome. Proc. Natl. Acad. Sci. USA 103: 5320-5325.

Davis, C. A., L. Grate, M. Spingola, and M. Ares, Jr., 2000 Test of intron predictions reveals novel splice sites, alternatively spliced mRNAs and new introns in meiotically regulated genes of yeast. Nucleic Acids Res. 28: 1700-1706.

Delneri, D., I. Colson, S. Grammenoudi, I. N. Roberts, E. J. Louis et al., 2003 Engineering evolution to study speciation in yeasts. Nature 422: 68-72.

Drinnenberg, I. A., D. E. Weinberg, K. T. Xie, J. P. Mower, K. H. Wolfe et al., 2009 RNAi in budding yeast. Science 326: 544 550.

Dujon, B., 2006 Yeasts illustrate the molecular mechanisms of eukaryotic genome evolution. Trends Genet. 22: 375-387.

Fewell, S. W., and J. L. Woolford, Jr., 1999 Ribosomal protein S14 of Saccharomyces cerevisiae regulates its expression by binding to RPS14B pre-mRNA and to 18S rRNA. Mol. Cell. Biol. 19: 826-834.

Gardner, E. J., Z. F. Nizami, C. C. Talbot, Jr., and J. G. Gall, 2012 Stable intronic sequence RNA (sisRNA), a new class of noncoding RNA from the oocyte nucleus of Xenopus tropicalis. Genes Dev. 26: 2550-2559.

Gardner, P. P., J. Daub, J. Tate, B. L. Moore, I. H. Osuch et al., 2010 Rfam: Wikipedia, clans and the "decimal" release. Nucleic Acids Res. 39: D141-D145.

Gietz, R. D., and R. H. Schiestl, 2007 High-efficiency yeast transformation using the LiAc/SS carrier DNA/PEG method. Nat. Protoc. 2: 31-34. 
Grund, S. E., T. Fischer, G. G. Cabal, O. Antunez, J. E. Perez-Ortin et al., 2008 The inner nuclear membrane protein Src1 associates with subtelomeric genes and alters their regulated gene expression. J. Cell Biol. 182: 897-910.

Guil, S., M. Soler, A. Portela, J. Carrere, E. Fonalleras et al., 2012 Intronic RNAs mediate EZH2 regulation of epigenetic targets. Nat. Struct. Mol. Biol. 19: 664-670.

Guldener, U., S. Heck, T. Fielder, J. Beinhauer, and J. H. Hegemann, 1996 A new efficient gene disruption cassette for repeated use in budding yeast. Nucleic Acids Res. 24: 2519-2524.

Hainer, S. J., J. A. Pruneski, R. D. Mitchell, R. M. Monteverde, and J. A. Martens, 2011 Intergenic transcription causes repression by directing nucleosome assembly. Genes Dev. 25: 29-40.

Hooks, K. B., and S. Griffiths-Jones, 2011 Conserved RNA structures in the non-canonical Hac1/Xbp1 intron. RNA Biol. 8: 552-556.

Hooks, K. B., D. Delneri, and S. Griffiths-Jones, 2014 Intron evolution in Saccharomycetaceae. Genome Biol. Evol. 6: 25432556.

Houseley, J., L. Rubbi, M. Grunstein, D. Tollervey, and M. Vogelauer, 2008 A ncRNA modulates histone modification and mRNA induction in the yeast GAL gene cluster. Mol. Cell 32: 685695.

Jeffares, D. C., T. Mourier, and D. Penny, 2006 The biology of intron gain and loss. Trends Genet. 22: 16-22.

Juneau, K., M. Miranda, M. E. Hillenmeyer, C. Nislow, and R. W. Davis, 2006 Introns regulate RNA and protein abundance in yeast. Genetics 174: 511-518.

Juneau, K., C. Nislow, and R. W. Davis, 2009 Alternative splicing of PTC7 in Saccharomyces cerevisiae determines protein localization. Genetics 183: 185-194.

Kahm, M., G. Hasenbrink, and H. Lichtenberg-Fraté, 2010 grofit: fitting biological growth curves with R. J. Stat. Softw. 33: 1-21.

Kim, Y. K., and V. N. Kim, 2007 Processing of intronic microRNAs. EMBO J. 26: 775-783.

Langmead, B., C. Trapnell, M. Pop, and S. L. Salzberg, 2009 Ultrafast and memory-efficient alignment of short DNA sequences to the human genome. Genome Biol. 10: R25.

Li, B., J. Vilardell, and J. R. Warner, 1996 An RNA structure involved in feedback regulation of splicing and of translation is critical for biological fitness. Proc. Natl. Acad. Sci. USA 93: 1596-1600.

Liao, Y., G. K. Smyth, and W. Shi, 2014 featureCounts: an efficient general purpose program for assigning sequence reads to genomic features. Bioinformatics 30: 923-930.

Louro, R., T. El-Jundi, H. I. Nakaya, E. M. Reis, and S. VerjovskiAlmeida, 2008 Conserved tissue expression signatures of intronic noncoding RNAs transcribed from human and mouse loci. Genomics 92: 18-25.

Louro, R., A. S. Smirnova, and S. Verjovski-Almeida, 2009 Long intronic noncoding RNA transcription: Expression noise or expression choice? Genomics 93: 291-298.

Lynch, M., 2002 Intron evolution as a population-genetic process. Proc. Natl. Acad. Sci. USA 99: 6118-6123.

Medina, E. M., G. W. Jones, and D. A. Fitzpatrick, 2011 Reconstructing the fungal tree of life using phylogenomics and a preliminary investigation of the distribution of yeast prion-like proteins in the fungal kingdom. J. Mol. Evol. 73: 116-133.

Mercer, T. R., M. E. Dinger, S. M. Sunkin, M. F. Mehler, and J. S. Mattick, 2008 Specific expression of long noncoding RNAs in the mouse brain. Proc. Natl. Acad. Sci. USA 105: 716-721.

Mitrovich, Q. M., B. B. Tuch, F. M. De La Vega, C. Guthrie, and A. D. Johnson, 2010 Evolution of yeast noncoding RNAs reveals an alternative mechanism for widespread intron loss. Science 330: 838-841.

Nakaya, H. I., P. P. Amaral, R. Louro, A. Lopes, A. A. Fachel et al., 2007 Genome mapping and expression analyses of human in- tronic noncoding RNAs reveal tissue-specific patterns and enrichment in genes related to regulation of transcription. Genome Biol. 8: R43.

Naseeb, S., and D. Delneri, 2012 Impact of chromosomal inversions on the yeast DAL cluster. PLoS One 7: e42022.

Nawrocki, E. P., D. L. Kolbe, and S. R. Eddy, 2009 Infernal 1.0: inference of RNA alignments. Bioinformatics 25: 1335-1337.

Neil, H., C. Malabat, Y. d'Aubenton-Carafa, Z. Xu, L. M. Steinmetz et al., 2009 Widespread bidirectional promoters are the major source of cryptic transcripts in yeast. Nature 457: 10381042.

Norris, M. G., S. C. Lovell, and D. Delneri, 2013 Characterization and prediction of haploinsufficiency using systems-level gene properties in yeast. G3 (Bethesda) 3: 1965-1977.

Ooi, S. L., D. A. Samarsky, M. J. Fournier, and J. D. Boeke, 1998 Intronic snoRNA biosynthesis in Saccharomyces cerevisiae depends on the lariat-debranching enzyme: intron length effects and activity of a precursor snoRNA. RNA 4: 1096-1110.

Parenteau, J., M. Durand, S. Veronneau, A. A. Lacombe, G. Morin et al., 2008 Deletion of many yeast introns reveals a minority of genes that require splicing for function. Mol. Biol. Cell 19: 1932-1941.

Parenteau, J., M. Durand, G. Morin, J. Gagnon, J. F. Lucier et al., 2011 Introns within ribosomal protein genes regulate the production and function of yeast ribosomes. Cell 147: 320 331.

Pircher, A., K. Bakowska-Zywicka, L. Schneider, M. Zywicki, and N. Polacek, 2014 An mRNA-derived noncoding RNA targets and regulates the ribosome. Mol. Cell 54: 147-155.

Plass, M., C. Codony-Servat, P. G. Ferreira, J. Vilardell, and E. Eyras, 2012 RNA secondary structure mediates alternative 3 'ss selection in Saccharomyces cerevisiae. RNA 18: 11031115.

Plocik, A. M., and C. Guthrie, 2012 Diverse forms of RPS9 splicing are part of an evolving autoregulatory circuit. PLoS Genet. 8: e1002620.

Preker, P. J., K. S. Kim, and C. Guthrie, 2002 Expression of the essential mRNA export factor Yra1p is autoregulated by a splicingdependent mechanism. RNA 8: 969-980.

Quinn, J. J., and H. Y. Chang, 2016 Unique features of long non-coding RNA biogenesis and function. Nat. Rev. Genet. 17: 47-62.

Rivas, E., and S. R. Eddy, 2000 Secondary structure alone is generally not statistically significant for the detection of noncoding RNAs. Bioinformatics 16: 583-605.

Rodriguez-Navarro, S., K. Strasser, and E. Hurt, 2002 An intron in the YRA1 gene is required to control Yra1 protein expression and mRNA export in yeast. EMBO Rep. 3: 438-442.

Rogic, S., B. Montpetit, H. H. Hoos, A. K. Mackworth, B. F. Ouellette et al., 2008 Correlation between the secondary structure of premRNA introns and the efficiency of splicing in Saccharomyces cerevisiae. BMC Genomics 9: 355.

Sasson, A., and T. P. Michael, 2010 Filtering error from SOLiD Output. Bioinformatics 26: 849-850.

Schneider, C., G. Kudla, W. Wlotzka, A. Tuck, and D. Tollervey, 2012 Transcriptome-wide analysis of exosome targets. Mol. Cell 48: 422-433.

Sidrauski, C., and P. Walter, 1997 The transmembrane kinase Ire $1 p$ is a site-specific endonuclease that initiates mRNA splicing in the unfolded protein response. Cell 90: 1031-1039.

Talhouarne, G. J., and J. G. Gall, 2014 Lariat intronic RNAs in the cytoplasm of Xenopus tropicalis oocytes. RNA 20: 1476-1487.

Torarinsson, E., Z. Yao, E. D. Wiklund, J. B. Bramsen, C. Hansen et al., 2008 Comparative genomics beyond sequence-based alignments: RNA structures in the ENCODE regions. Genome Res. 18: 242-251. 
Trapnell, C., L. Pachter, and S. L. Salzberg, 2009 TopHat: discovering splice junctions with RNA-Seq. Bioinformatics 25: 1105-1111.

Wang, J., P. J. Smith, A. R. Krainer, and M. Q. Zhang, 2005 Distribution of SR protein exonic splicing enhancer motifs in human protein-coding genes. Nucleic Acids Res. 33: 50535062.

Wu, J., D. Delneri, and R. T. O'Keefe, 2012 Non-coding RNAs in Saccharomyces cerevisiae: What is the function? Biochem. Soc. Trans. 40: 907-911.
Xu, Z., W. Wei, J. Gagneur, F. Perocchi, S. Clauder-Munster et al., 2009 Bidirectional promoters generate pervasive transcription in yeast. Nature 457: 1033-1037.

Yin, Q. F., L. Yang, Y. Zhang, J. F. Xiang, Y. W. Wu et al., 2012 Long noncoding RNAs with snoRNA ends. Mol. Cell 48: 219-230.

Zhang, Y., X. O. Zhang, T. Chen, J. F. Xiang, Q. F. Yin et al., 2013 Circular intronic long noncoding RNAs. Mol. Cell 51: 792-806.

Communicating editor: L. M. Steinmetz 


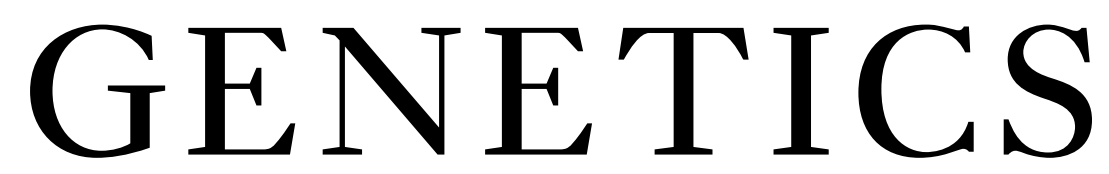

Supporting Information www.genetics.org/lookup/suppl/doi:10.1534/genetics.115.185363/-/DC1

\section{Novel Intronic RNA Structures Contribute to Maintenance of Phenotype in Saccharomyces cerevisiae}

Katarzyna B. Hooks, Samina Naseeb, Steven Parker, Sam Griffiths-Jones, and Daniela Delneri 


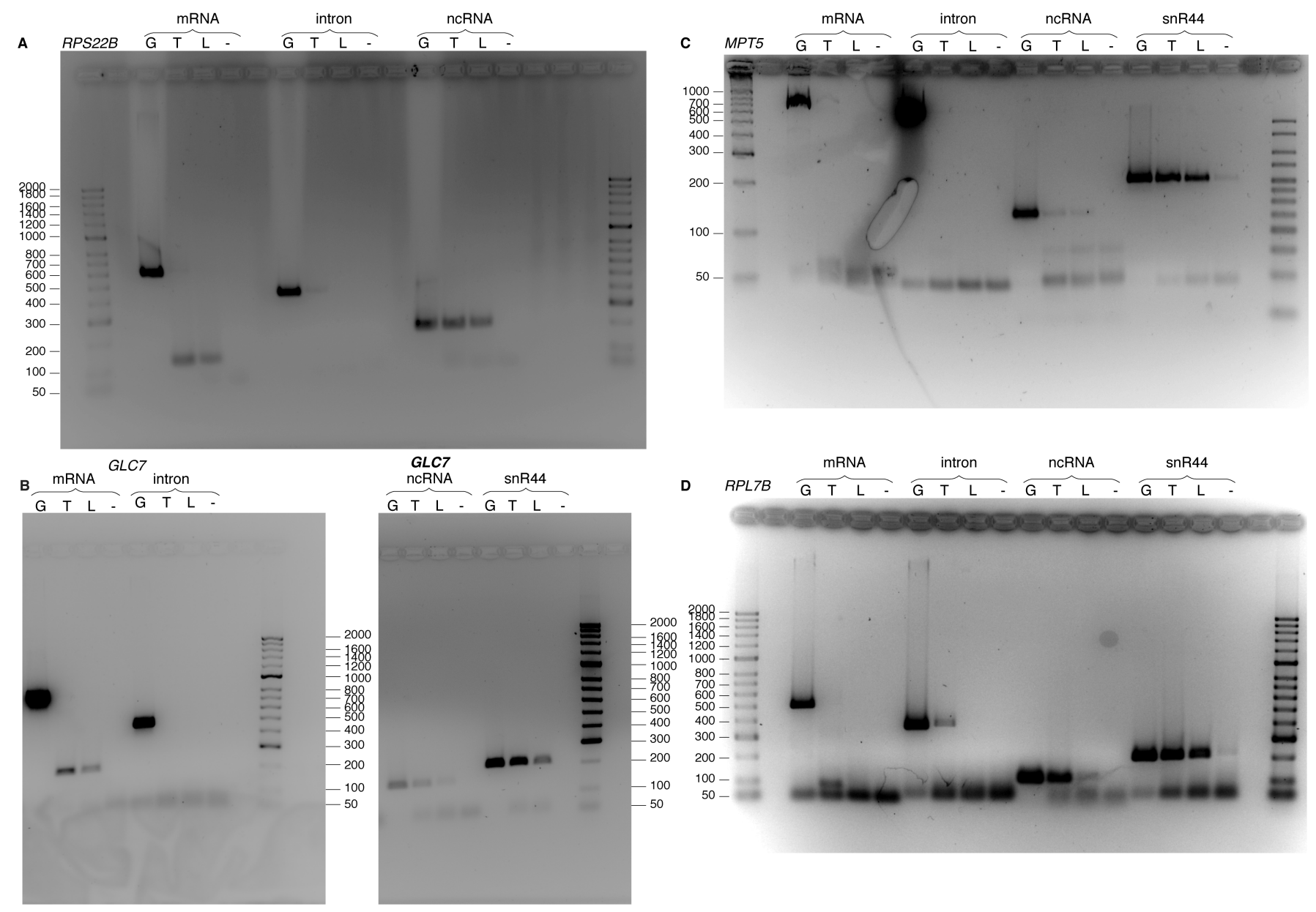

Figure S1. RT-PCR confirmation of ncRNA expressed from introns. Products for: (A) RPS22B, (B) GLC7, (C) MPT5, (D) RPL7B. Lane designations for DNA templates: G, Genomic DNA; T, total cDNA; L, low molecular weight enriched cDNA; (-), no template negative control. Except GLC7 ncRNA, which was run on a separate gel, for each gene brightness and contrast applied equally across the entire gel image. 


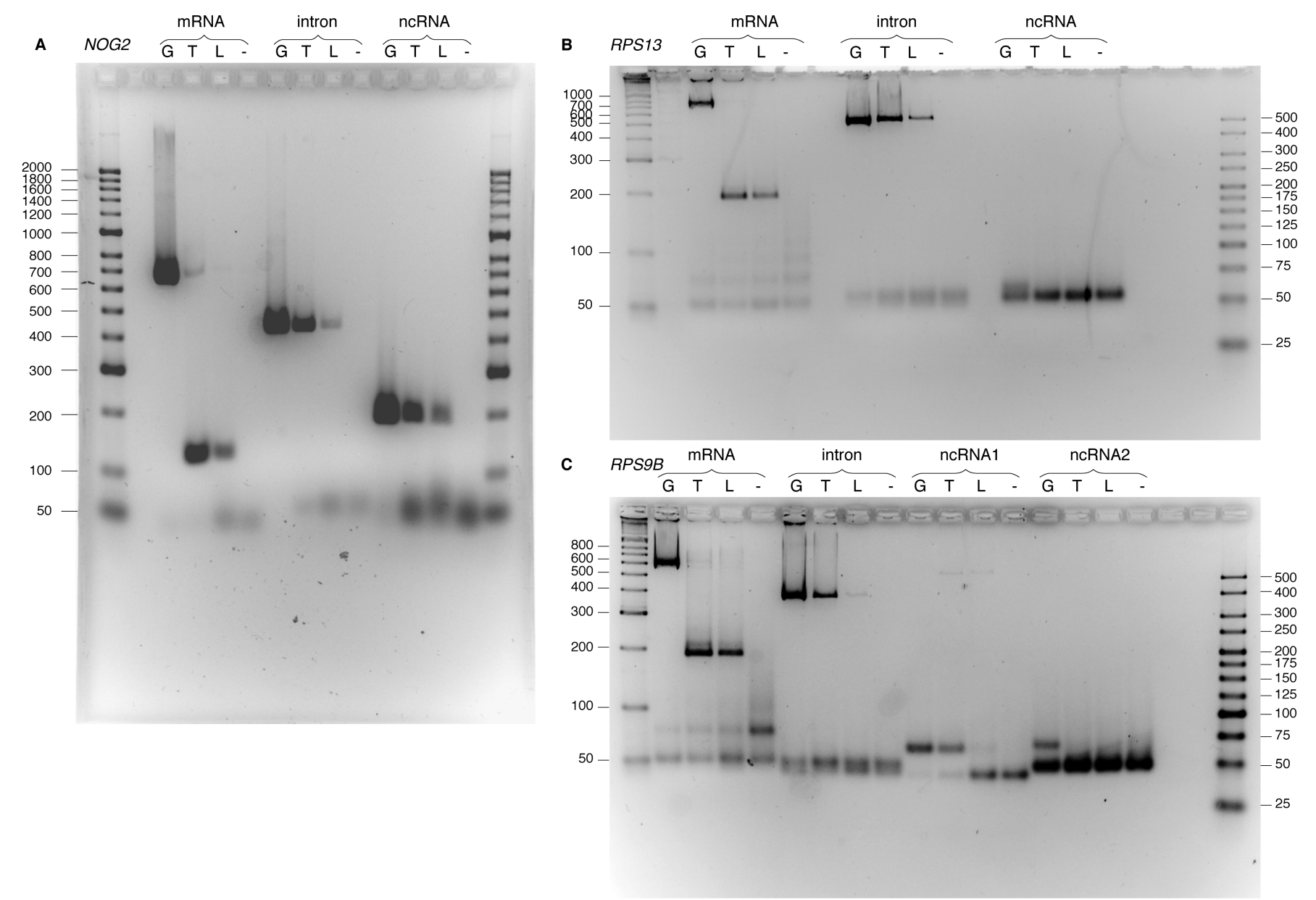

Figure S2. RT-PCR confirmation of intron expression accompanied by complete mRNA splicing. Products for: (A) NOG2, (B) RPS13, (C) RPS9B. Lane designations for DNA templates: G, genomic DNA; T, total cDNA; L, low molecular weight enriched cDNA; (-), no template negative control. Brightness and contrast were applied equally across each gel image. 


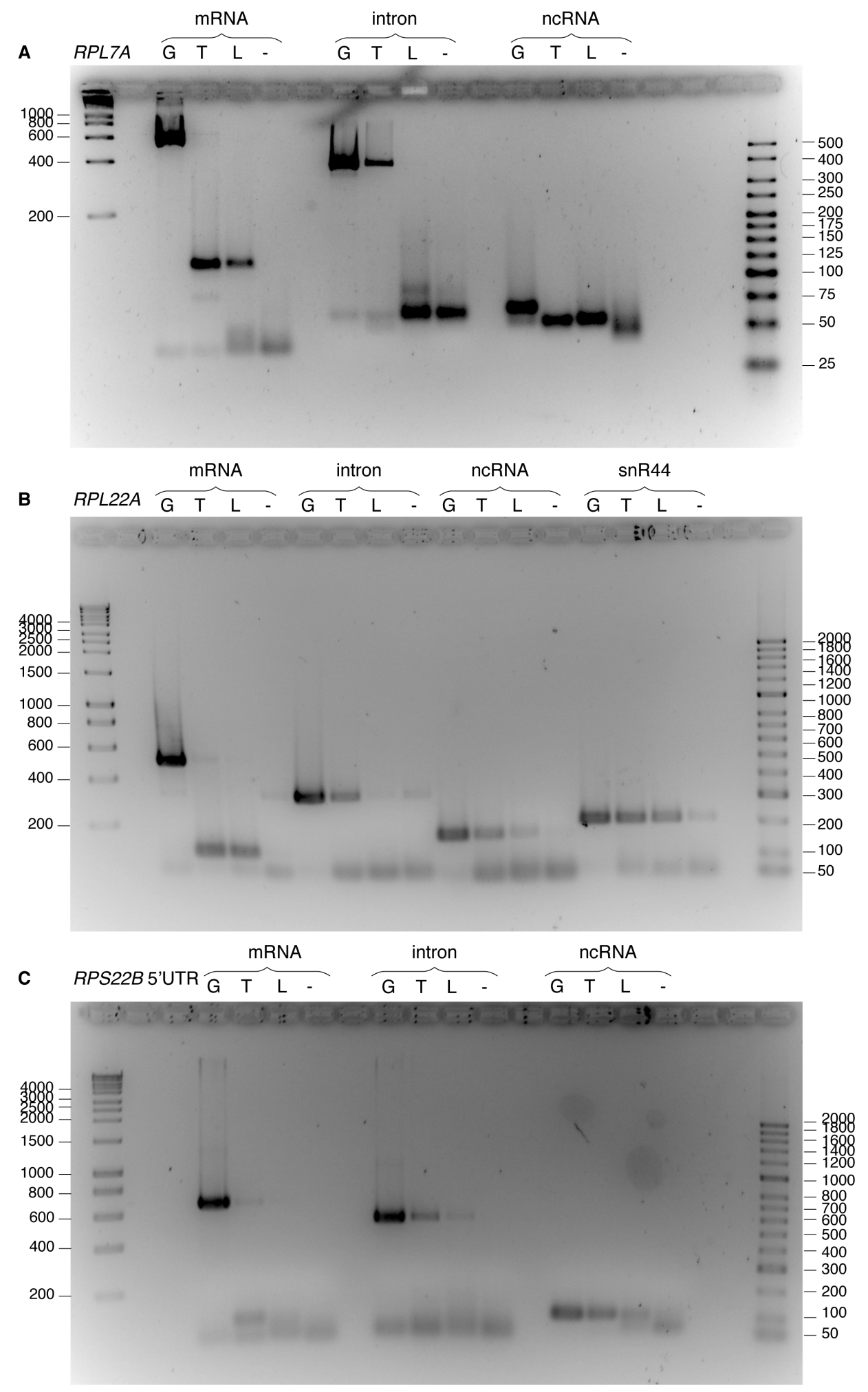

Figure S3. RT-PCR confirmation of intron expression accompanied by complete mRNA splicing. Products for: (A) RPL7A, (B) RPL22A, (C) RPS22B $5^{\circ} \mathrm{UTR}$. Lane designations for DNA templates: G, genomic DNA; T, total cDNA; L, low molecular weight enriched cDNA; $(-)$, no template negative control. Brightness and contrast were applied equally across each gel image. 

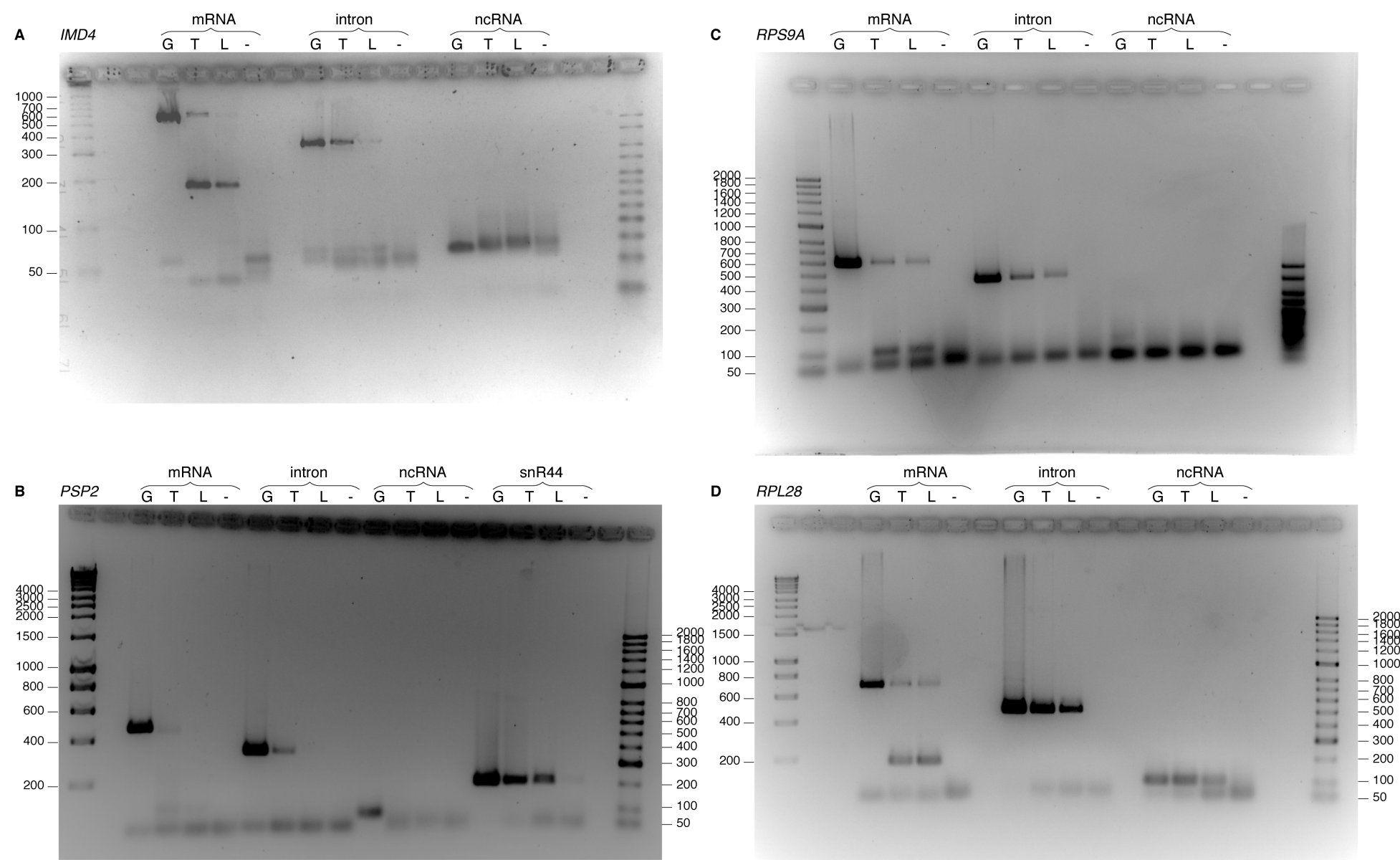

Figure S4. RT-PCR confirmation of alternative splicing. Products for: (A) IMD4, (B) PSP2, (C) RPS9A, (D) RPL28. Lane designations for DNA templates: G, genomic DNA; T, total cDNA; L, low molecular weight enriched cDNA; $(-)$, no template negative control. Brightness and contrast was applied equally across each gel image. 

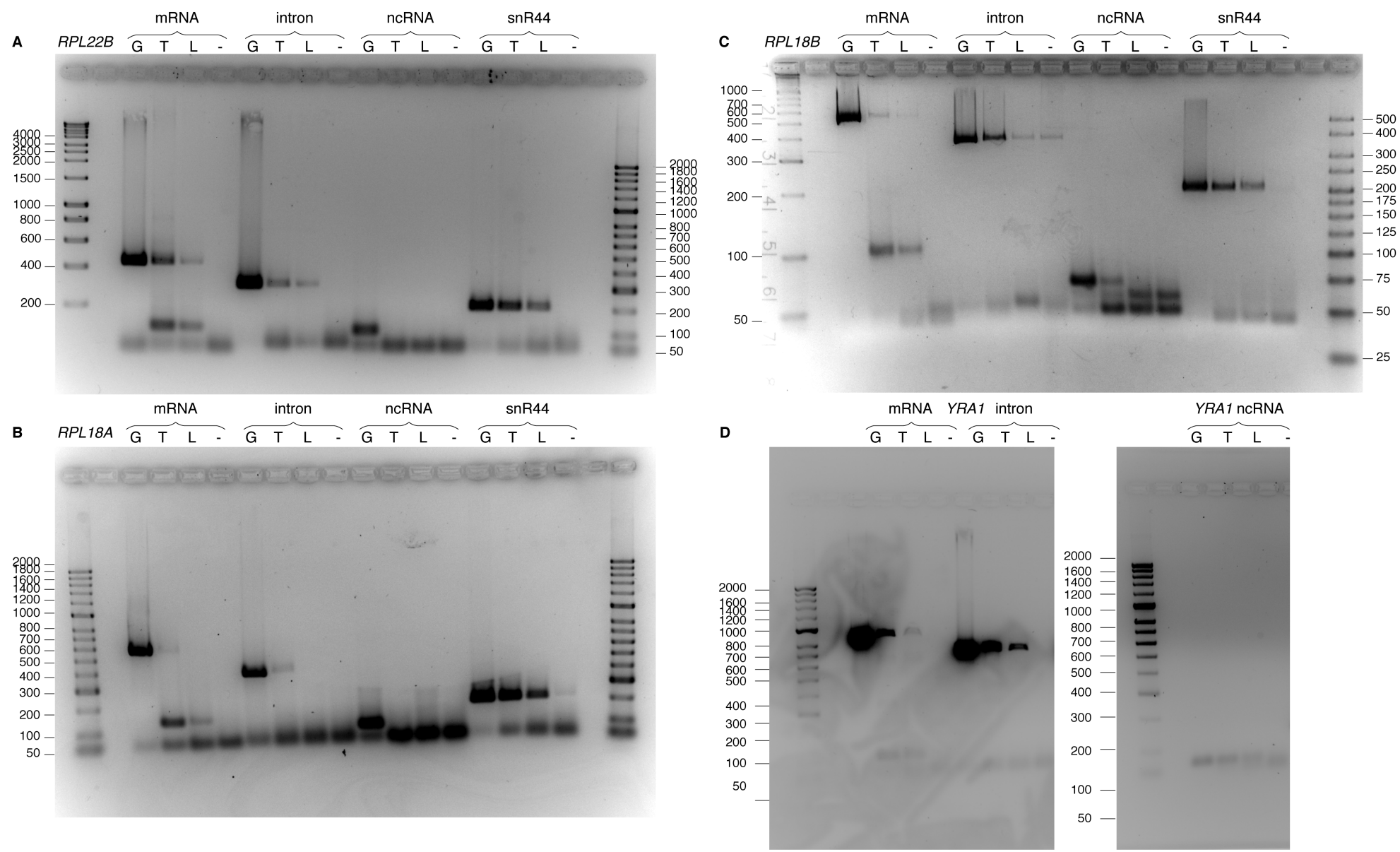

Figure S5. RT-PCR confirmation of alternative splicing. Products for: (A) RPL22B, (B) RPL18A, (C) RPL18B, (D) YRA1. Lane designations for DNA templates: G, Genomic DNA; T, total cDNA; L, low molecular weight enriched cDNA; (-), no template negative control. Except YRA1 ncRNA, which was run on a separate gel, for each gene brightness and contrast applied equally across the entire gel image. 


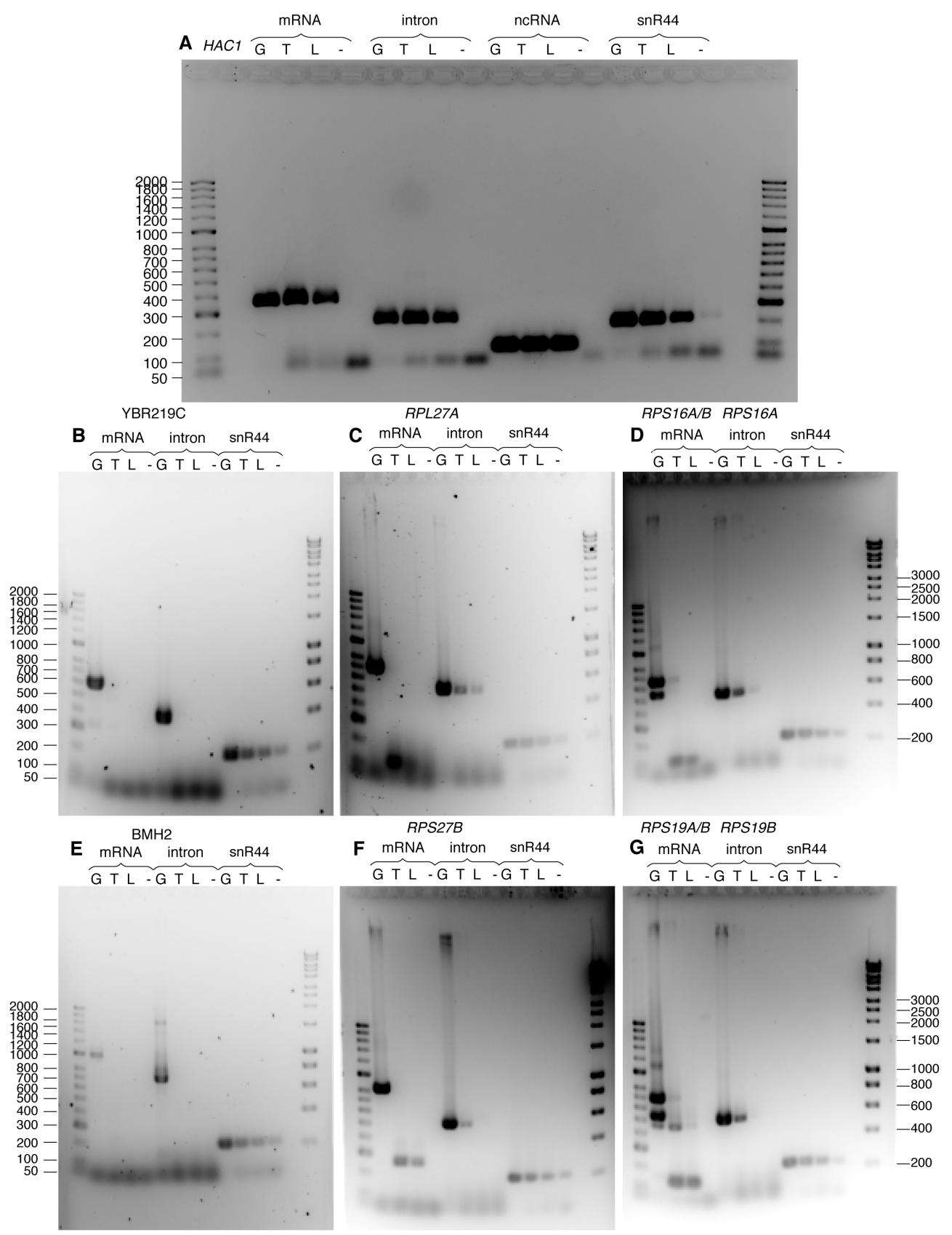

Figure S6. Control RT-PCRs confirming mRNA splicing status and intron expression. PCR products for genes: (A) HAC1, (B) YBR219C, (C) RPL27, (D) RPS16A/B, (E) BMH2, (F) $R P S 27 B$, (G) RPS19A/B. Lane designations for DNA templates: $\mathrm{G}$, Genomic DNA; T, total cDNA; L, low molecular weight enriched cDNA; (-), no template negative control. Brightness and contrast was applied equally across each gel image. For RPS16A and RPS19B designed mRNA primers also hybridize with the paralogous genes RPS16B and RPS19A giving two products from genomic DNA template. 
A

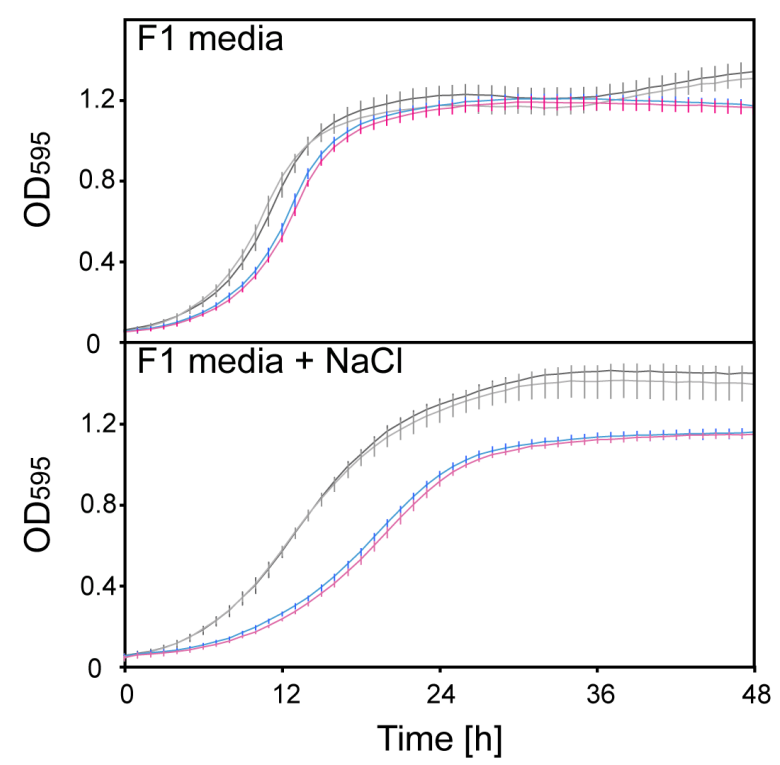

C

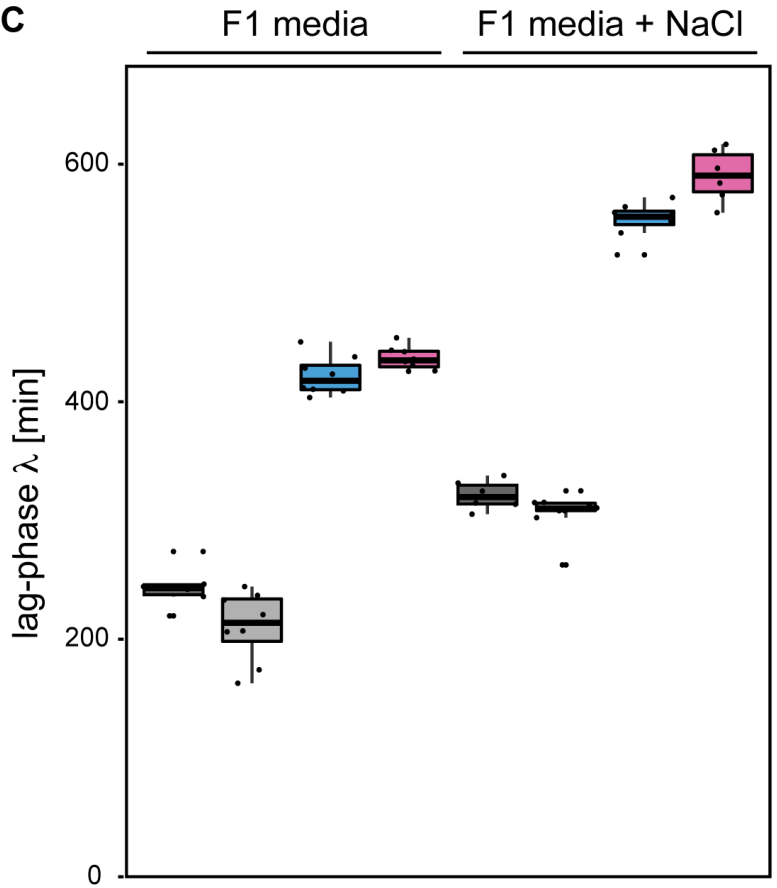

B

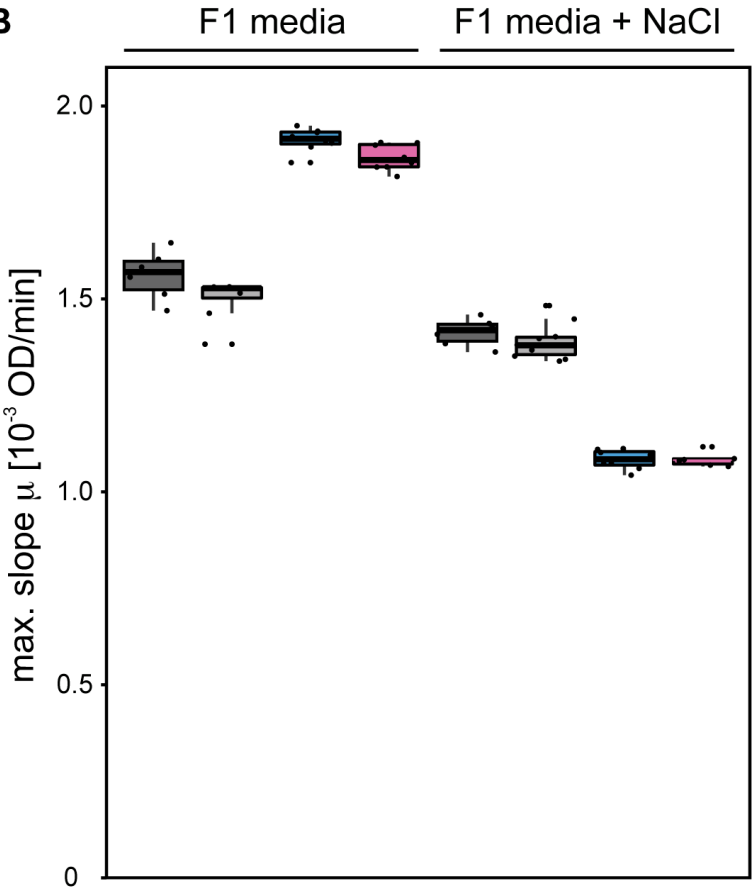

D

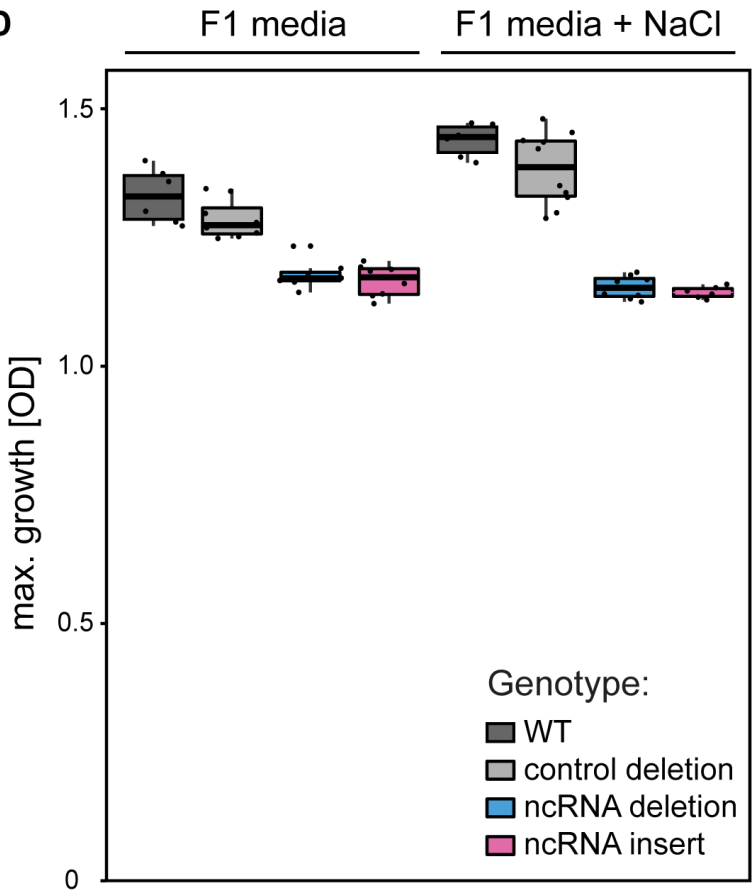

Figure S7. Growth of GLC7 mutants in $\mathrm{F} 1$ and $\mathrm{F} 1+\mathrm{NaCl}$ media. (A) Growth curves made by plotting average OD measurements for each mutant taken every 60 minutes with bars representing standard deviation. (B) Maximum slope of a growth curve for each mutant. (C) Lag-phase time estimated from growth curves. (D) Maximal growth for each mutant. Mutants are labelled as displayed on the legend in the bottom right corner. Data presented in (B)-(D) was obtained by growth curve fitting with $\mathrm{R}$ package grofit. 


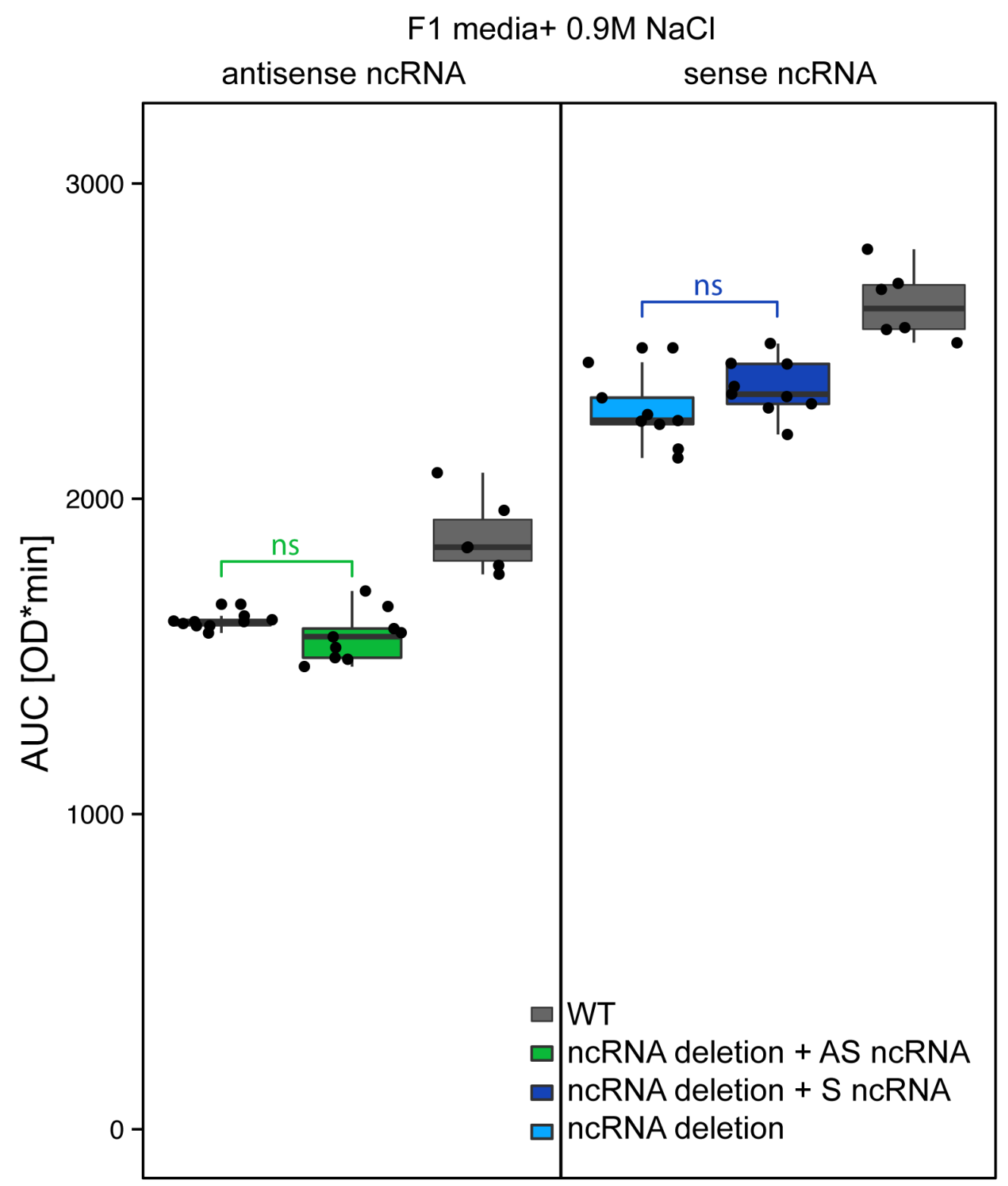

Figure S8. Phenotypic effect of reintroducing ncRNA into GLC7 ncRNA deletion mutant. ncRNA in sense and antisense orientation was inserted into modified pRS315 vector under the TDH3 promoter. Both plasmids were transformed into GLC7 ncRNA mutants to check their ability to rescue phenotypic defect. Two separate growth experiments were performed in $\mathrm{F} 1+0.9 \mathrm{M} \mathrm{NaCl}$ media for each orientation of the ncRNA(AS: antisense; S: sense). Values in the box plots present the means of the area under the growth curve (AUC) as determined by R pracma package. The differences between ncRNA deletion mutant with and without ncRNA expressed from the vector are not significant (t-test, $\mathrm{p}>0.05$ ). 
Table S1. List of fungal genomes used for BLAST searches.

\begin{tabular}{|c|c|c|c|c|}
\hline Organism & Strain & Source & Ver & $\begin{array}{l}\text { Reference } \\
\text { [PMID] }\end{array}$ \\
\hline Aspergillus fumigatus & Af293 & Broad Inst. & 1 & 16372009 \\
\hline Aspergillus nidulans & FGSC A4 & Broad Inst. & 1 & 16372000 \\
\hline Aspergillus oryzae & RIB40 & NITE & 1 & 16372010 \\
\hline Aspergillus terreus & NIH2624 & Broad Inst. & 1 & - \\
\hline Botryotinia fuckeliana & B05.10 & Broad Inst. & 1 & - \\
\hline Candida albicans & SC5314 & Stanford Uni & 2 & 15123810 \\
\hline Candida glabrata & CBS138 & Genolevures & 1 & 15229592 \\
\hline Chaetomium globosum & CBS 148.51 & Broad Inst. & 1 & - \\
\hline Coccidioides immitis & H538.4 & Broad Inst. & 1 & - \\
\hline Coprinopsis cinerea & okayama7\#130 & Broad Inst. & 1 & - \\
\hline Cryptococcus neoformans & serA H99 & Broad Inst. & 1 & - \\
\hline Debaryomyces hansenii & CBS767 & Genolevures & 1 & 15229592 \\
\hline Eremothecium gossypii & ATCC 10895 & Biozentrum & 1 & 15001715 \\
\hline Fusarium graminearum & PH-1 & Broad Inst. & 3 & 17823352 \\
\hline Fusarium verticillioides & 7600 & Broad Inst. & 3 & - \\
\hline Histoplasma capsulatum & G217B & WashU & 1 & - \\
\hline Kluyveromyces lactis & NRRL Y-1140 & Genolevures & 1 & 15229592 \\
\hline Lachancea kluyveri & NRRL Y-12651 & Stanford Uni & 1 & - \\
\hline Lachancea waltii & NCYC 2644 & Broad Inst. & 1 & 15004568 \\
\hline Magnaporthe grisea & $70-15$ & Broad Inst. & 5 & 15846337 \\
\hline Naumovozyma castellii & NRRL Y-12630 & Stanford Uni & 1 & 15229592 \\
\hline Neurospora crassa & OR74A & Broad Inst. & 7 & 12712197 \\
\hline Parastagonospora nodorum & SN15 & Broad Inst. & 1 & - \\
\hline Phanerochaete chrysosporium & RP-78 & DOE Joint Genome & 1 & 15122302 \\
\hline Rhizopus oryzae & RA 99-880 & Broad Inst. & 1 & - \\
\hline $\begin{array}{l}\text { Saccharomyces bayanus var. } \\
\text { uvarum }\end{array}$ & $623-6 C$ & Stanford Uni & 1 & 15229592 \\
\hline Saccharomyces cerevisiae & S288c & SGD & 1 & 9169864 \\
\hline Saccharomyces kudriavzevii & IFO 1802 & Stanford Uni & 1 & 15229592 \\
\hline Saccharomyces mikatae & IFO 1815 & Stanford Uni & 1 & 15229592 \\
\hline Saccharomyces paradoxus & NRRL Y-17217 & Stanford Uni & 1 & 15229592 \\
\hline Schizosaccharomyces japonicus & yFS275 (SJ5) & Broad Inst. & 1 & 21511999 \\
\hline Schizosaccharomyces pombe & $972 \mathrm{~h}$ & Sanger Inst. & 1 & 11859360 \\
\hline Sclerotinia sclerotiorum & 1980 & Broad Inst. & 1 & - \\
\hline Trichoderma reesei & QM6a & DOE Joint & 2 & 18454138 \\
\hline Uncinocarpus reesii & 1704 & Broad Inst. & 1 & - \\
\hline Ustilago maydis & 521 & Broad Inst. & 1 & 17080091 \\
\hline Yarrowia lipolytica & CLIB122 & Genolevures Cons. & 1 & 15229592 \\
\hline
\end{tabular}


File S1: List of RNAz, CMfinder and EvoFold predictions for each intron. In columns listed are: all significant structures predicted by RNAz with their $p$-values, all significant scores for EvoFold together with the highest score for each intron, the number of distinctive significant motives predicted by CMfinder together with $r$ score and folding energy for each prediction. (.xls, $93 \mathrm{~KB}$ )

Available for download as a .xls file at:

http://www.genetics.org/lookup/suppl/doi:10.1534/genetics.115.185363/-/DC1/FileS1.xls 
File S2: Primer sequences and probes used in the study. List contains forward and reversed primers used for generating and confirming deletion mutants, RT-PCR, real time PCR and probes used for Northern. (.xls, $69 \mathrm{~KB})$

Available for download as a .xls file at:

http://www.genetics.org/lookup/suppl/doi:10.1534/genetics.115.185363/-/DC1/FileS2.xls 
File S3: Expression of introns and coding sequences of host genes [RPKM] and the average percentile from exosome targets data (CRAC) for each gene. (.xls, $168 \mathrm{~KB}$ )

Available for download as a .xls file at:

http://www.genetics.org/lookup/suppl/doi:10.1534/genetics.115.185363/-/DC1/FileS3.xls 